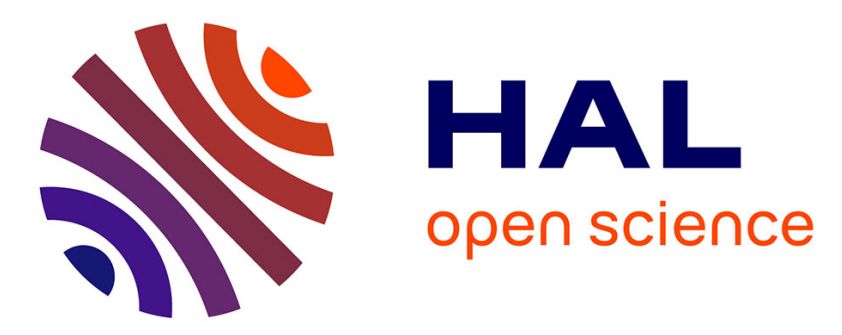

\title{
Analytical modeling of the transient ablation of a 3D $\mathrm{C} / \mathrm{C}$ composite
}

\author{
J. Lachaud, Y. Aspa, Gérard Vignoles
}

\section{To cite this version:}

J. Lachaud, Y. Aspa, Gérard Vignoles. Analytical modeling of the transient ablation of a 3D C/C composite. International Journal of Heat and Mass Transfer, 2017, 115, pp.1150-1165. 10.1016/j.ijheatmasstransfer.2017.06.130 . hal-01761299

\section{HAL Id: hal-01761299 \\ https://hal.science/hal-01761299}

Submitted on 8 Apr 2018

HAL is a multi-disciplinary open access archive for the deposit and dissemination of scientific research documents, whether they are published or not. The documents may come from teaching and research institutions in France or abroad, or from public or private research centers.
L'archive ouverte pluridisciplinaire HAL, est destinée au dépôt et à la diffusion de documents scientifiques de niveau recherche, publiés ou non, émanant des établissements d'enseignement et de recherche français ou étrangers, des laboratoires publics ou privés. 


\title{
Analytical modeling of the transient ablation of a 3D $\mathrm{C} / \mathrm{C}$ composite
}

\author{
J. Lachaud ${ }^{\mathrm{a}, *}$, Y. Aspa ${ }^{\mathrm{a}, * *}$, Gérard L. Vignoles ${ }^{\mathrm{a}}$ \\ ${ }^{a}$ Université Bordeaux 1 \\ Laboratoire des Composites ThermoStructuraux (LCTS) \\ 3, Allée de La Boétie, 33600 Pessac, France
}

\begin{abstract}
Experimental studies have shown that dense $\mathrm{C} / \mathrm{C}$ composites feature a complex surface ablation behavior under oxidation conditions. Their effective reactivity is not an additive property with respect to the intrinsic reactivities of their components. Typical surface roughness patterns progressively appear and the effective reactivity increases with time until it reaches a steady state value. In a previous work, the steady-state behavior of a 3D C/C composite has been modeled and explained. Here, the work has been extended to address the transient regime behavior. The surface roughness evolution and the effective reactivity are computed versus time. The model is validated by comparison with experimental data. These theoretical results provide for the first time a comprehensive understanding of the complex ablation process occurring at the surface of dense $\mathrm{C} / \mathrm{C}$ composites.

Keywords:

$\mathrm{C} / \mathrm{C}$ composite, ablation, surface roughness, reactivity, modeling
\end{abstract}

${ }^{*}$ Currently C la Vie/PPME, University of New Caledonia, Nouméa, New Caledonia.

${ }^{* *}$ Currently Airbus Safran Launchers, Saint Médard en Jalles, France. 


\section{Introduction}

Carbon/carbon $(\mathrm{C} / \mathrm{C})$ composites are used in demanding thermostructural applications [1-3] - e.g. heat shields of space vehicles, nozzles of rocket thrusters, aircraft and formula one brake disks, diverters of nuclear fusion reactors [4]. They are made of porous carbon fiber reinforcements densified with carbon matrix. At the atomic scale, reinforcements and matrices are both composed of hexagonal graphene planes linked by weak van der Waals bonds. This layered structure is called turbostratic carbon or pseudographite [5]. Graphene is the thermodynamically stable phase of carbon under standard conditions and up to theits sublimation temperature. The remarkable properties of graphene [6] translate at the composite scale. C/C composites do not melt and they display excellent mechanical properties up to $3000 \mathrm{~K}$. One major drawback for thermostructural applications, however, is the high reactivity of graphene plane edges with molecular oxygen at temperatures above $500 \mathrm{~K}[7,8]$. Often used in the $1000 \mathrm{~K}$ temperature range in oxidizing atmospheres (brakes, rocket thrusters) or even up to the sublimation temperature (heat shields, nuclear reactors), $\mathrm{C} / \mathrm{C}$ composites are subject to oxidation and sublimation in typical thermostructural applications. Oxidation and sublimation lead to a progressive surface recession, called ablation, that needs to be controlled. The ablative behavior of $\mathrm{C} / \mathrm{C}$ composites has been extensively studied since their first production, about 60 years ago [9-34]. Three major observations have been made : (1) the composite geometric reactivity is not an additive property with respect to the

intrinsic reactivities of its components (reinforcement and matrix), (2) the reactivity is found to increase with time until it reaches a steady state value, 
and (3) a typical surface roughness progressively appears during oxidation.

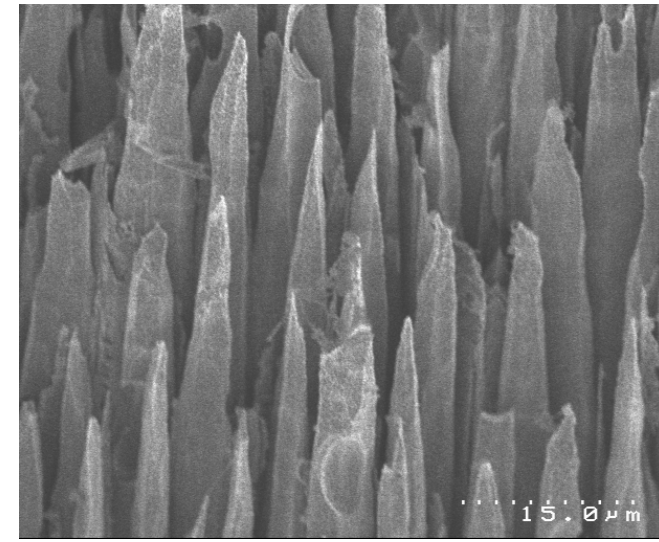

a- Laboratory oven, air at $900 \mathrm{~K}$

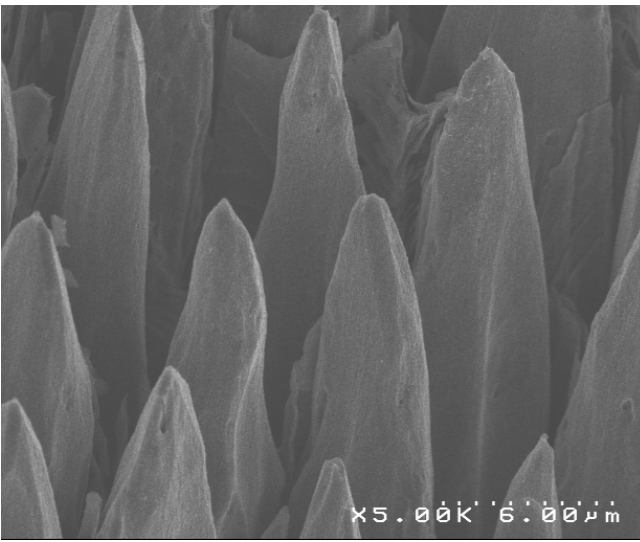

c- Plasma jet, air at $2500 \mathrm{~K}$

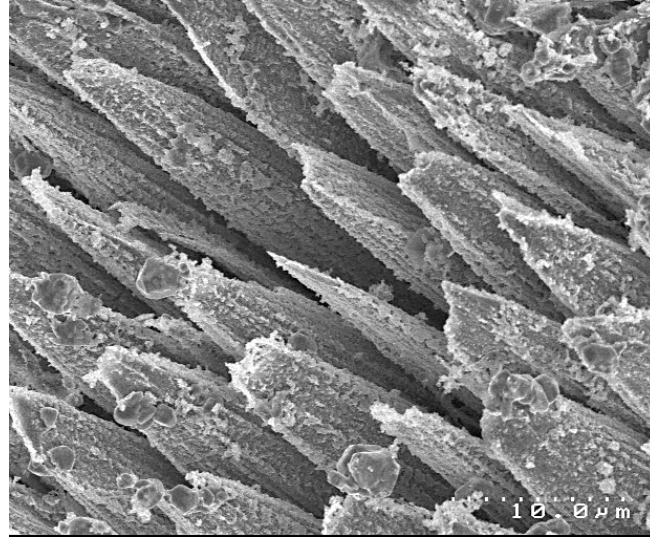

b- Rocket nozzle, water vapor at $1500 \mathrm{~K}$

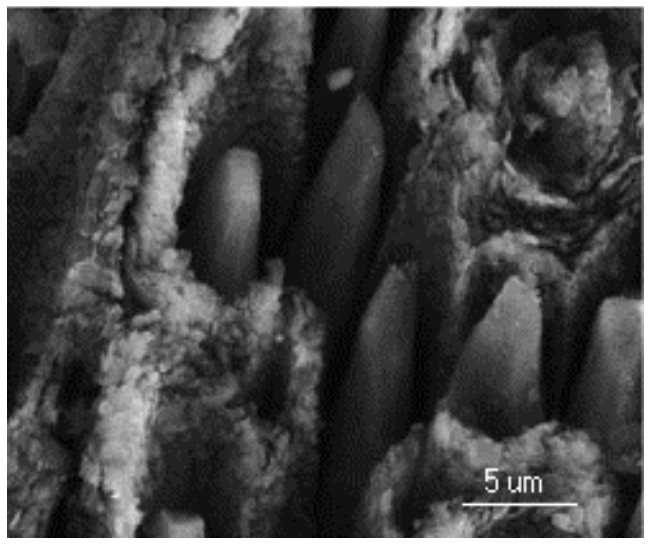

d- Nuclear reactor, sublimation at $3500 \mathrm{~K}$

Figure 1: Scanning electron micrographs at the fiber scale of ablated $\mathrm{C} / \mathrm{C}$ composites.

At the fiber scale, typical needle shapes are described throughout the literature for different $\mathrm{C} / \mathrm{C}$ composites and ablation conditions. In figure 1, scanning electron micrographs are presented for four diverse conditions. The first oneneedle shape (fig. 1a) has been obtained by oxidation under dry air at $898 \mathrm{~K}$ in a laboratory oven [30]. The second one shape results is 


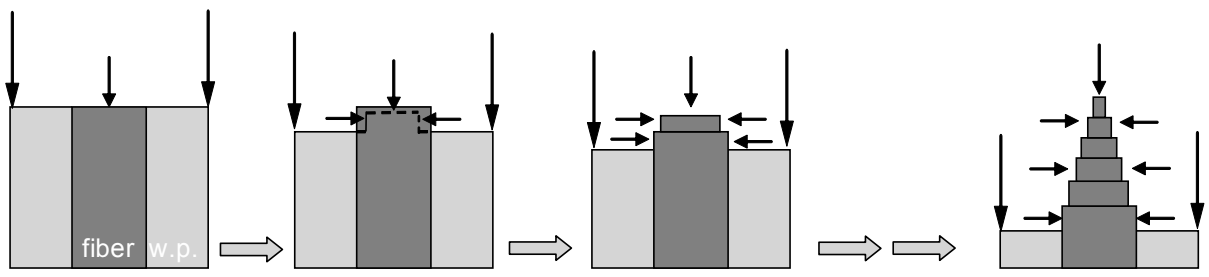

a) Reactive regime (fast diffusion). The weak phase is more reactive. Its initial recession rate is faster. The fiber is uncoverred and oxidized from the sides. The fiber takes a needle shape.The surface area of the fiber increases. In steady state, its effective reactivity is equal to the reactivity of the weak phase.

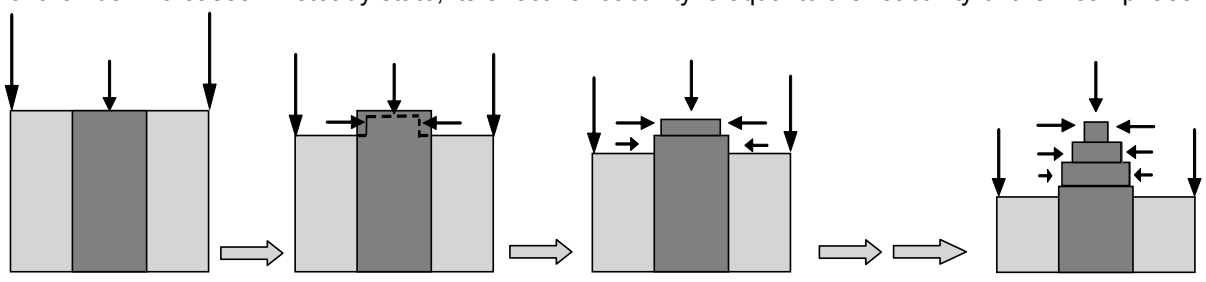

b) Mixed regime (moderate diffusive limitation). Oxygen, which diffuses from the boundary layer, is partialy consummed by the fiber. The oxygen concentration decreases from the fiber tip to the surface of the weak phase. The fiber takes an ogival shape. The effective reactivity is a complicated function of the oxygen diffusion coefficient and of the fiber and weak phase reactivities.

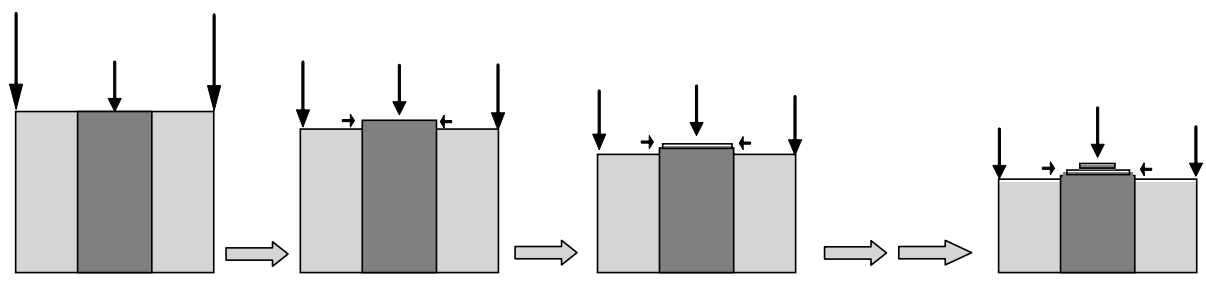

c) Diffusive regime (fast reaction). Both ablation rates are fast compared to diffusive transport. Oxygen transport limits the surface recession rate for both phases. The effective oxidation rate is not a function of the reactivities of the phases. It is only a function of the diffusion coefficient.

Figure 2: Illustration of the oxidation behavior at the fiber scale

from oxidation by water vapor at $1500 \mathrm{~K}$ in a rocket nozzle [35]. The third one shape was taken after air plasma jet testing of a space vehicle thermal

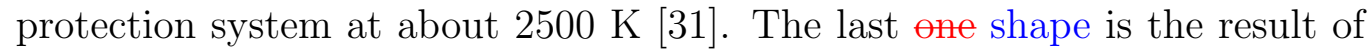
sublimation in the diverter of the International Thermonuclear Experimental Reactor (ITER) at a temperature estimated between 3000 and $3500 \mathrm{~K}$ [36]. In these four applications, the ablation conditions are significantly different but the same typical needle shape is observed. It is interesting to notice that 
similar behaviors are observed in the oxidation and sublimation regimes. This is easily explained as rigorously equivalent mathematical models can be derived to describe sublimation and heterogeneous oxidation reactions [37]. For most materials, carbon matrices are more reactive than carbon fibers [30]. For some $\mathrm{C} / \mathrm{C}$ composites, the bulk matrix properties are very similar to the fiber properties, at least in term of oxidative behavior. Only a weak transition phase, called interface [20] or interphase [30], and lying between the fibers and the bulk matrix, is more reactive. It is clearly observed in figure 1-d that the matrix height - hence its resistance to sublimation - is similar to that of the carbon fibers but that there is a more reactive phase between them. In what follows, we will use the expression "weak phase", to describe the most reactive phase, be it the matrix itself or only a thin interphase.

The mechanism leading to the typical pointed fiber shape has previously been modeled and explained [4, 27, 32]. In figure 2-a, we propose an illustration with a fiber embedded in a more reactive weak carbon phase. The weak phase oxidizes and recesses faster. The sides of the fiber are exposed. The fiber tip as well as the sides of the fibers are oxidized, leaving after an elementary time step a smaller protruding cylinder (in dashed lines in figure a). Step by step, the fiber progressively takes the well known needle shape. Of course, the stepwise description is only used here for illustration. This mechanism happens at the microscopic scale and produces smooth needle shapes from homogeneous cylindrical carbon fibers [32]. A steady state is reached when the surface area of the exposed fiber is such that it compensates for its 
lower reactivity, with the following balance being reached

$$
k_{f} S_{f}=k_{w p} S_{p}
$$

where $k_{f}$ and $k_{w p}$ are the fiber and weak phase reactivities, and $S_{f}$ and $S_{p}$ are respectively the surface developed by the fiber and its projected surface, that is, its initial surface when the surface was flat. A rigorous mathematical derivation is provided in reference [4]. Another interesting fact is that the exact fiber shape slightly varies from one application to another. Of course, the nature of the fiber plays a role when comparing different materials. The same material was used for both oxidation tests (figure 1-a and 1-c) and it appears that the fiber shape is rather ogival in the arc jet test conditions. It is a well know fact that oxidation rates increase with temperature and that diffusion becomes a limiting process [3], even at the fiber length scale $[27,38]$. In this case, fibers take ogival shapes rather than needle shapes [4], we have shown, as pictured in figure 2-b. When diffusion is fully limiting, then no surface roughness should be observed in theory, as represented in figure 2-c, but to our knowledge, no observation of flat ablated surfaces has been reported so far for $\mathrm{C} / \mathrm{C}$ composites.

We previously developed and validated a multiscale analytical model to predict the steady-state surface roughness and effective oxidation rate of a 3D C/C composite from the intrinsic reactivity of its components [4]. In this work, we extend the steady-state model to the transient regime (section 2), provide an analytical solution (section 3), and validate the transient model by comparison with experimental data (section 4). Finally, we conclude in section 5 . 


\section{Presentation of the multiscale modeling approach}

This work is an extension of a previous one [4]. The same physical assumptions (material geometry and external flow conditions) and base mathematical model will be used. For convenience, we provide a synthetic summary in this section. Figure 3 is used to recall the architecture of the material, the multiscale modeling approach, and the ablation conditions.

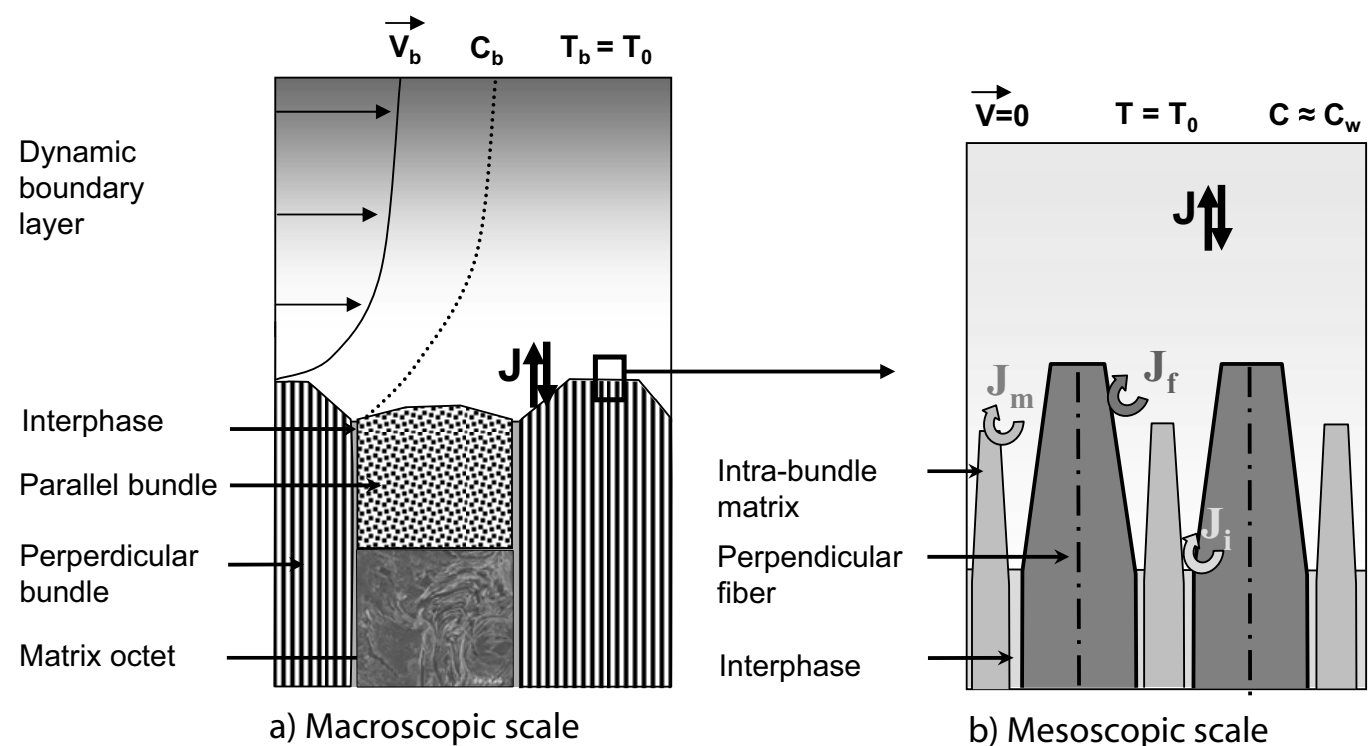

Figure 3: Modeling strategy : sketch of the ablation phenomena and of the surface roughness of a $3 \mathrm{D} \mathrm{C} / \mathrm{C}$ in transient regime

\subsection{Description of the $3 D C / C$ composite}

The studied material is a 3D C/C composite, made from a 3D ex-PAN (PolyAcryloNitrile) carbon fiber preform and a pitch-based graphitized carbon matrix. It is a heterogeneous multiscale material. Straight yarns, made of several thousands of parallel fibers, are densified with carbon matrix into 
unidirectional bundles. The bundles are orthogonally fit together into a pattern repeated by translation on a cubic lattice. This macrostructure leads to a network of parallelepipedic macropores (located near each node of the lattice), which are filled with matrix and are called octets.

\subsection{Modeling approach and assumptions}

The modeling approach needs to follow the multiscale architecture of the composite. Two changes of scale are needed to infer the composite behavior from the properties of its components. First, the bundle properties are inferred from that of their components (fibers, interphase, intra-yarn matrix) at mesoscopic scale (figure 3-b). Second, the composite behavior is obtained from the bundles, inter-bundle matrix, and matrix octet properties at the macroscopic scale (figure 3-a).

A simple reaction/diffusion model has been chosen for this transient multiscale analysis of ablation. It considers a reactive material immersed in a continuum flow. Thermal gradients and advection close to the wall are neglected. Diffusion is the main mode of mass transport of oxidant in the boundary layer. The ablation reaction is assumed to be first-order. Theses hypotheses have been shown to be valid in the laboratory oven oxidation conditions used in the steady-state study $[4,30]$. They will be reused in this work in the transient regime.

\subsection{Mathematical description of the reaction/diffusion ablation model}

Let $J_{e}$ be the molar impinging flux of reactant per unit surface unit given by a first order kinetic law : $J_{e}=k_{e} . C$, with $k_{e}$ the oxidation rate of the material element $e$ (namely, one of the phases; e.g. a carbon fiber) and $C$ 
the reactant concentration (e.g. oxygen concentration). Mass conservation of the reactant in the fluid phase writes:

$$
\frac{\partial C}{\partial t}+\nabla \cdot(-D \nabla C)=0
$$

where $D$ (in $\left.m^{2} \cdot s^{-1}\right)$ is the diffusion coefficient of the reactant in the bulk fluid phase.

Boundary conditions relative to the model domain are :

- On the boundary layer top: $C=C_{0}$;

- At the fluid/solid interface the molar oxidation rate $J_{e}$ above the solid element $e$ writes:

$$
J_{e}=(-D \nabla C) \cdot \mathbf{n}=-k_{e} C
$$

where $\mathbf{n}$ is the surface normal;

- Periodicity on lateral boundaries.

Gasification processes lead to recession of material surfaces. The macroscopic motion of a given surface $S$ can be interpreted as an advancing wavefront. The surface position is commonly described in cartesian coordinates by the following scalar equation

$$
S(x, y, z, t)=0
$$

such that the function $S$, which possesses almost everywhere first order partial derivatives, acquires nonzero values at all points not lying on the interface [39]. The function $S$ satisfies the differential equation

$$
\frac{\partial S}{\partial t}+\mathbf{v}_{\mathbf{e}} \cdot \nabla S=0
$$


where $\mathbf{v}_{\mathbf{e}}=\Omega_{e} J_{e} \mathbf{n}$ is the surface local normal velocity of the surface, with $\Omega_{e}$ the solid molar volume of $e$ [39]. The coupled resolution of equations 2,3 , and 4 provides the evolution of the surface roughness and effective reactivity of the composite with time.

\section{Analytical solution}

Analytical upscaling models are presented in this section. As explained in the previous section, two upscaling steps are necessary : the first one at the mesoscopic scale (subsection 3.1) and the second one at the macroscopic scale (subsection 3.2). The validity of the scale separation approach is studied $a$ posteriori in subsection 3.3.

\subsection{Mesoscopic scale}

As represented in figure 3, there are three components at the mesoscopic scale: perpendicular bundles, parallel bundles, and carbon matrix octets. Matrix octets properties are homogeneous and their properties are experimentally obtained [30]. The objective of this subsection is to develop a model for the bundles, made of thousands of carbon fibers and impregnated with carbon matrix. Inside the bundles, the fibers are assumed parallel to each other and to the bundle axis, homogeneous, isotropic, axi-symmetrical, and surrounded by a homogeneous weak phase (the interphase) as represented in figure 4-a. In this subsection, we develop an analytical model for perpendicular bundles; the case of parallel bundles is treated in Appendix A.

We will start by recalling steady state results [4]. Scanning electron micrography (SEM) observations have shown that the bulk matrix height is lower than that of the fibers $\left(h_{f}\right)$ and higher than that of the weak-phase 


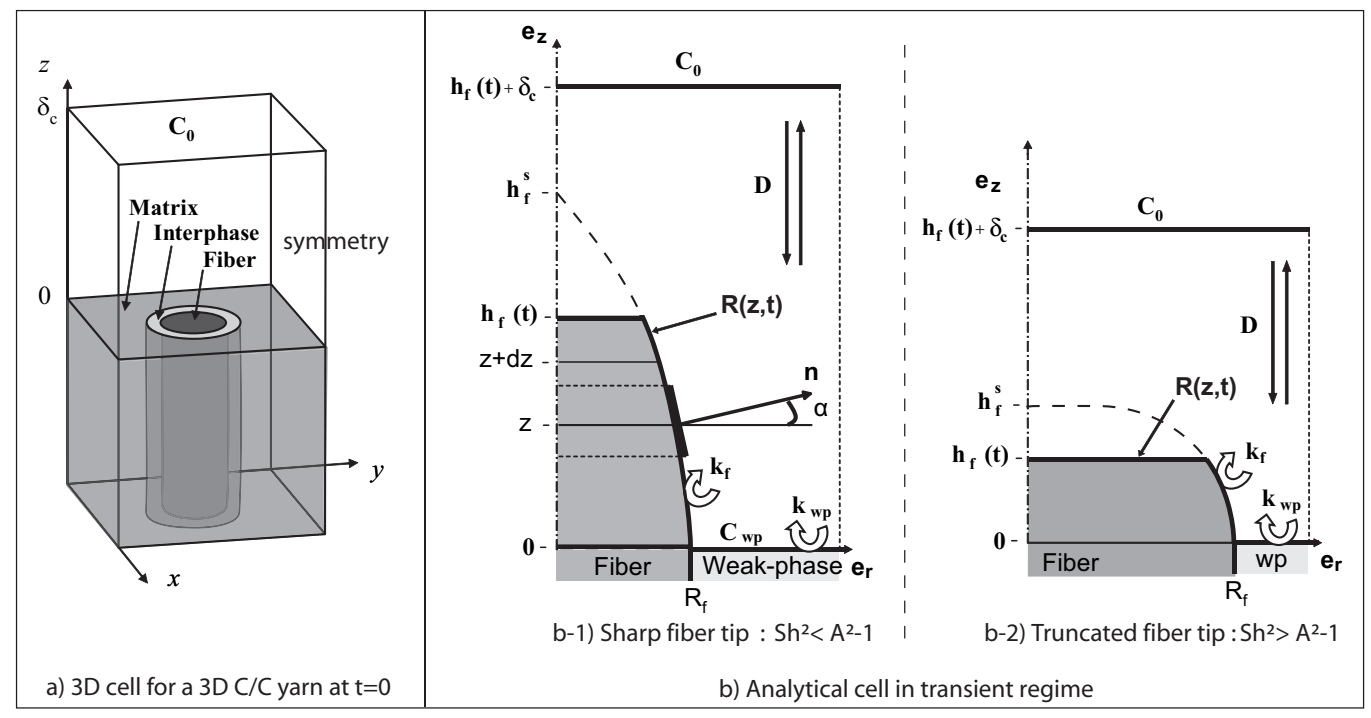

Figure 4: Illustration of the elementary pattern and of the proposed model in transient regime

$(h=0)$. As a result, under the vertical mass transfer hypothesis, the intrayarn matrix displays no influence on the fibers and interphase behaviors. Moreover, the latter determines the overall yarn behavior [4]. The problem is more conveniently treated in cylindrical coordinates, as represented in figure 4-b. The surface of the fiber can be described by the following equation

$$
S_{f}(r, \theta, z, t)=r-R(z, t)=0
$$

where $\mathrm{R}$ is a function defining the surface of the fiber as a function of elevation $(z)$ and time $(t)$. In steady state the time dependency is removed such that $R(z, t)=R(z)$. In this case, we have previously shown [4] with two resolution methods that the dimensionless fiber surface $\widetilde{R}(\widetilde{z})=R(\widetilde{z}) / R_{f}$ is given by the positive part of 


$$
\widetilde{R}(\widetilde{z})=S h^{-1}\left(\sqrt{A^{2}-(1+S h \widetilde{z})^{2}}-\sqrt{A^{2}-1}\right)+1
$$

with $\widetilde{z} \in\left[0,(A-1) / S h\left[. R_{f}\right.\right.$ is the fiber radius, $\widetilde{z}=z / R_{f}, S h=R_{f} k_{w p} / D$ is a Sherwood number, and $A=\left(k_{w p} \Omega_{w p}\right) /\left(k_{f} \Omega_{f}\right)$ is the ratio of reactivity $(k)$ and molar volume $(\Omega)$ eontrast between of the fibers (subscript $f$ ) and the weak phase (subscript $w p$ ). We can introduce a convenient auxiliary variable $\tilde{u}=1+S h \tilde{z}$, so that :

$$
\widetilde{R}(\widetilde{z})=S h^{-1}\left(\sqrt{A^{2}-\tilde{u}^{2}}-\sqrt{A^{2}-1}\right)+1
$$

with $\tilde{u} \in[1 ; A]$.

For $S h<\sqrt{A^{2}-1}$, the fiber tip is sharp and $\widetilde{h_{f}^{s}}$ writes

$$
\widetilde{h_{f}^{s}}=\sqrt{S h^{-2}+2 S h^{-1} \sqrt{A^{2}-1}-1}-S h^{-1}
$$

or:

$$
\widetilde{u_{f}^{s}}=\sqrt{A^{-2}-\left(\sqrt{A^{2}-1}-S h\right)^{2}}
$$

In figures 4-b-1, the steady state shape is represented in dashed line (the solid line is for transient regime and will be studied next). For $S h \geq \sqrt{A^{2}-1}$, the fiber tip is always truncated as pictured figure 4-b-2, and its steady-state height $\widetilde{h_{f}^{s}}=h_{f}^{s} / R_{f}$ is limited to $(A-1) /$ Sh, i.e. $\widetilde{u_{f}^{s}}=A$.

It has been shown that the values of $C_{0}$ and of $\delta$ (thickness of the concentration boundary layer) have no influence on the final surface-roughness features [4]. However, they affect the ablation velocity as well as the duration of the transient regime. The concentration gradient in the concentration boundary layer is vertical in all regimes (this is verified by directdetailed numerical simulation in appendix A). The concentration on the fiber plateau 
flat top in transient regime is then given by [4] :

$$
C_{e f f}(t)=C_{0} \frac{1}{1+\frac{k_{y}^{e f f}(t) \delta}{D}}
$$

where $k_{y}^{e f f}(t)$ is the effective reactivity of the yarn (fiber and matrix) in transient regime at the position $h_{f}(t)$. Let $k_{y}^{e f f}(t)$ be determined. According to the unidirectional gradient hypothesis, the concentration on the weak phase is given by [4] :

$$
C_{w p}(t)=C_{e f f}(t) \frac{1}{1+\frac{k_{w p} h_{f}(t)}{D}}
$$

The molar flux by surface unit consumed by the weak phase is then

$$
J_{w p}(t)=k_{w p} C_{e f f}(t) \frac{1}{1+\frac{k_{w p} h_{f}(t)}{D}}
$$

Considering the transient regime configuration, the part of the fiber between $\widetilde{R}=1$ and $\widetilde{R}(\widetilde{h}(t))$ has the same recession velocity as the weak phase, that is, its consumed molar flux by surface unit projected on matrix plane, $J_{f}$, is equal to $J_{w p}$. The molar volumes of fiber and weak phase are equal for the material of the study. The molar flux by surface unit consumed by the fibers on the plateau flat top is simply given by

$$
J_{f}(t)=k_{f} C_{e f f}(t)
$$

The total vertical flux of the composite unit cell is the sum of a contribution from the fiber top, with flux $J_{f}$, from the fiber lateral surface (between $\widetilde{R}=1$ and $\widetilde{R}(\widetilde{h}(t))$ ), with flux $J_{w p}$, and from the weak phase. The relative proportions of these contributions are respectively $\frac{\pi R\left(h_{f}\right)^{2}}{S_{t}}$, 


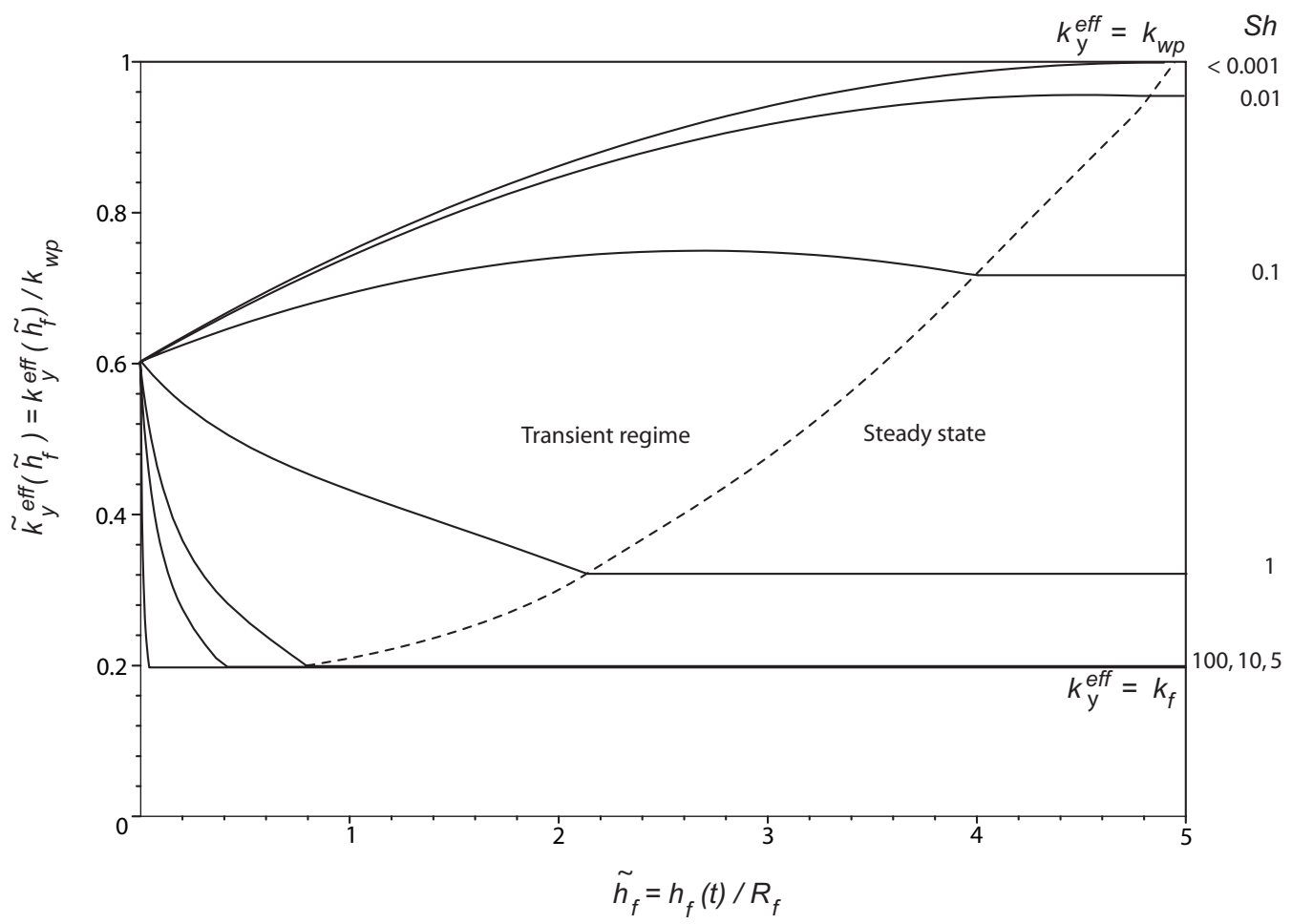

Figure 5: Normalized effective reactivity versus fibers height $\left(A=5, \widetilde{S}_{t}=2\right)$.

$\frac{\pi R_{f}^{2}-\pi R\left(h_{f}\right)^{2}}{S_{t}}$ and $\left(1-\frac{\pi R_{f}^{2}}{S_{t}}\right)$, where $S_{t}$ is the total surface of the cell :

$$
J_{t}(t)=\frac{\pi R\left(h_{f}\right)^{2}}{S_{t}} J_{f}(t)+\frac{\pi R_{f}^{2}-\pi R\left(h_{f}\right)^{2}}{S_{t}} J_{w p}(t)+\left(1-\frac{\pi R_{f}^{2}}{S_{t}}\right) J_{w p}(t)
$$

or :

$$
J_{t}(t)=\frac{\pi R\left(h_{f}\right)^{2}}{S_{t}} J_{f}(t)+\left(1-\frac{\pi R\left(h_{f}\right)^{2}}{S_{t}}\right) J_{w p}(t)
$$

Defining $\widetilde{S}_{t}=S_{t} /\left(\pi R_{f}^{2}\right)$ and $\widetilde{R}=R / R_{f}$, this becomes:

$$
J_{t}(t)=\frac{\widetilde{R}\left(h_{f}\right)^{2}}{\widetilde{S}_{t}} J_{f}(t)+\left(1-\frac{\widetilde{R}\left(h_{f}\right)^{2}}{\widetilde{S}_{t}}\right) J_{w p}(t)
$$


Combining this expression with equations (11-14), one obtains the normalized effective reactivity of the yarn as a function of the normalized height $\widetilde{h_{f}}=h_{f}(t) / R_{f}$.

$$
\widetilde{k}_{y}^{e f f}\left(\widetilde{h_{f}}\right)=\frac{\left(\widetilde{R}\left(\widetilde{h_{f}}\right)\right)^{2}}{A \widetilde{S}_{t}}+\left(1-\frac{\left(\widetilde{R}\left(\widetilde{h_{f}}\right)\right)^{2}}{\widetilde{S}_{t}}\right) \frac{1}{1+S h \widetilde{h_{f}}}
$$

with $\widetilde{k}_{y}^{\text {eff }}=k_{y}^{\text {eff }} / k_{w p}$.,$\widetilde{S}_{t}=S_{t} /\left(\pi R_{f}^{2}\right)$ (where $S_{t}$ is the total surface of the cell), $\widetilde{R}=R / R_{f}$. This can be rewritten as :

$$
\widetilde{k}_{y}^{e f f}\left(\widetilde{u_{f}}\right)=\frac{1}{\widetilde{u_{f}}}+\frac{\left(\widetilde{R}\left(\widetilde{h_{f}}\right)\right)^{2}}{\widetilde{S}_{t}}\left(\frac{1}{A}-\frac{1}{\widetilde{u_{f}}}\right)
$$

$\widetilde{k}_{y}^{e f f}$ is represented in figure 5 for values of $S h$ ranging from reactionlimited to diffusion-limited regime for $A=5$ and $\widetilde{S}_{t}=2$. The initial effective reactivity is equal to the arithmetic average of the fiber and the matrix reactivities. Once ablation starts, the weak phase progressively uncovers the fiber, which increases its exposed area. In reaction-limited regime, the concentration in the whole fluid phase being constant and equal to $C_{0}$, the effective reactivity of the yarn progressively tends to that of the weak phase. The steady state is reached when $k_{y}^{e f f}=k_{w p}$. When diffusional effects become non negligible, the weak phase is partly protected by the fiber. In diffusion limited-regime $\left(S h>\sqrt{A^{2}-1}\right)$, this protection progressively increases until the effective reactivity reaches the strongest phase reactivity (here the fiber) in steady state. Between these limiting cases, the reactivity may increase due to the reaction-limitation effect, or decrease due to diffusive limitation, or first increase and then decrease, as seen e. $g$. for $S h=0.1$. 
The value of $h_{f}$ as a function of time can be inferred from $k_{y}^{e f f}$. The growth of $h_{f}$ as a function of time is given by the difference between the vertical recession velocities of fibers $\left(v_{f}\right)$ and weak phase $\left(v_{w p}\right)$ :

$$
\frac{d h_{f}}{d t}(t)=v_{w p}-v_{f}=C_{w p} k_{w p} \Omega_{w p}-C_{e f f} k_{f} \Omega_{f}
$$

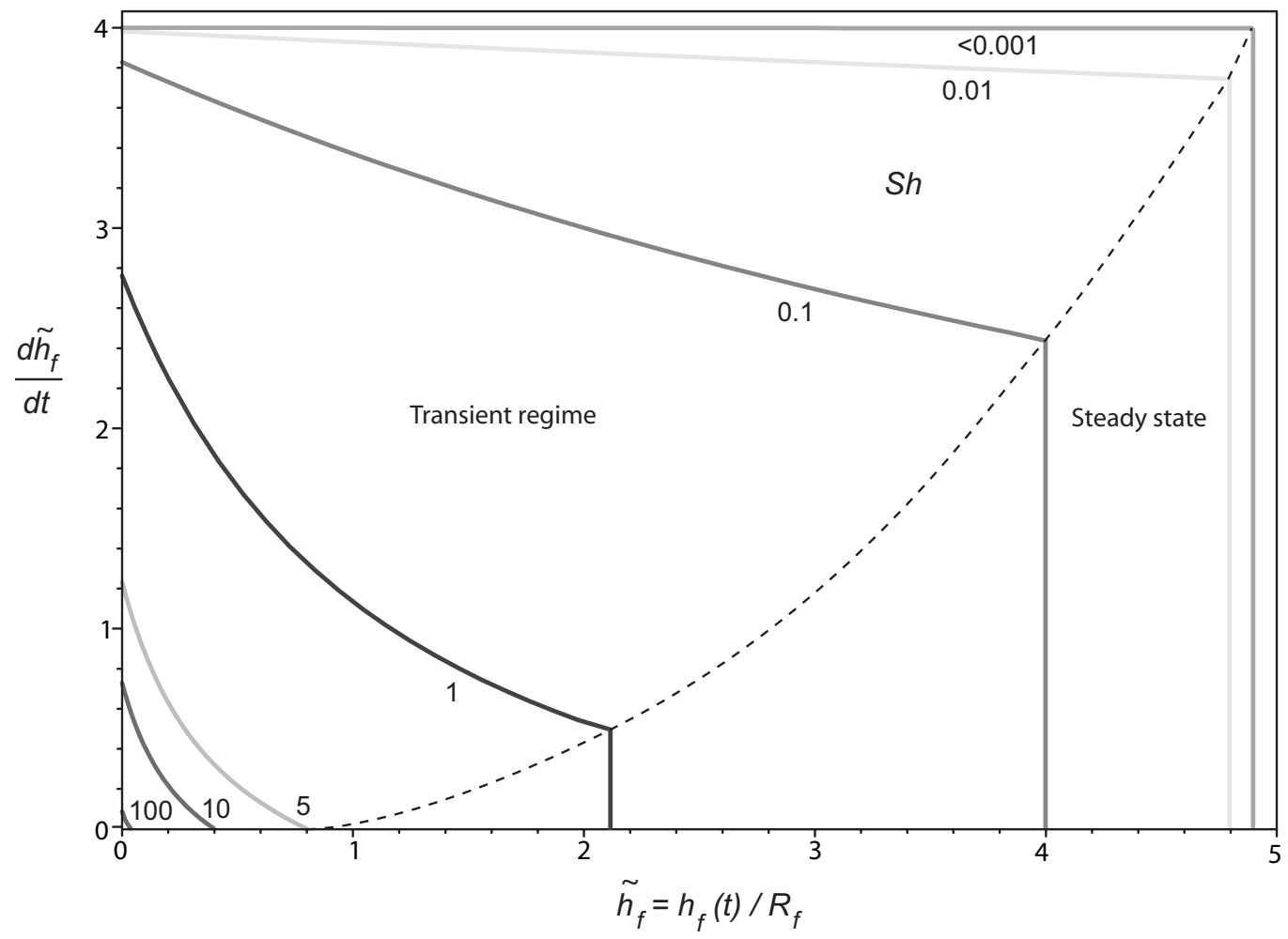

Figure 6: Velocity of fiber height growth as a function of fiber height $\left(A=5, \widetilde{S}_{t}=2\right)$, according to eqs. (18) and (21).

Substituting equations (11-12) in equation (20) and rewriting the latter in dimensionless form, one obtains the following differential equation for $\widetilde{h_{f}}(t)$ 


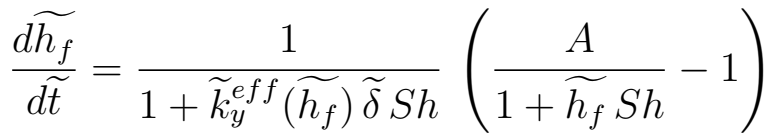

with $\widetilde{t}=t / \tau$ (where $\tau=R_{f} /\left(C_{0} k_{f} \Omega_{f}\right)$ is a characteristic time of the problem), $\widetilde{\delta}=\delta / R_{f}$, and the initial condition for integration being $\widetilde{h_{f}}(t=$ $0)=0$. Eq. (21) can be rewritten as:

$$
\frac{d \widetilde{u_{f}}}{d \widetilde{t}}=\frac{1}{S h^{-1}+\widetilde{\delta} \widetilde{k_{y}^{e f f}\left(\widetilde{u_{f}}\right)}}\left(\frac{A}{\widetilde{u_{f}}}-1\right)
$$

The right hand side of equation (21), which is a complicated eomplex function of $\widetilde{h_{f}}(t)$, is represented in figure 6 for different values of $S h$ and for $A=5, \widetilde{S}_{t}=2$ and $\widetilde{\delta}=0.75$ (note that $\widetilde{k}_{y}^{\text {eff }}$ in eq. (21) is computed using eq. (18)). Of course, the growth velocity of $\widetilde{h_{f}}$ (noted $\widetilde{h_{f}}$ ) is null by definition in steady state, as a stationary height is reached and maintained. In reaction-limited regime $(S h<0.001)$, the growth velocity is constant and given by $A-1$ in transient regime. When $S h$ increases, diffusive effects impact roughness growth. An increase of $\widetilde{h_{f}}$ leads to a protection of the weak phase by the fiber and reduced roughness growth rate with time. The initial values of $\widetilde{h_{f}}$ on in figure 6 are linked to the initial effective reactivity of the weak phase.

To explicit $\widetilde{h_{f}}$ as a function of time, one has to solve differential equation (21). Eq. (22) can be solved more conveniently. The time to reach a given height $\widetilde{h_{f}}=\left(\widetilde{u_{f}}-1\right) / S h$ is evaluated by the following integration :

$$
t=\int_{1}^{\widetilde{u_{f}}}\left(S h^{-1}+\widetilde{\delta} \widetilde{k}_{y}^{e f f}\left(\widetilde{u_{f}}\right)\right) \frac{\widetilde{u_{f}}}{A-\widetilde{u_{f}}} d \widetilde{u_{f}}
$$


The integration is presented in Appendix B. The result is:

$$
\begin{aligned}
t= & -\left(S h^{-1}+\widetilde{\delta} A^{-1}\right) \ln \left(\frac{A-\widetilde{u_{f}}}{A-1}\right)-\ldots \\
& \ldots-\left(S h^{-1}+\widetilde{\delta} \frac{2 A^{2}-{\widetilde{u_{f}^{s}}}^{2}}{A \widetilde{S}_{t} S h^{2}}\right)\left(\widetilde{u_{f}}-1\right)+\frac{\widetilde{\delta}}{3 A \widetilde{S}_{t} S h^{2}}\left({\widetilde{u_{f}}}^{3}-1\right)-\ldots \\
& \ldots-\widetilde{\delta} \frac{S h-\sqrt{A^{2}-1}}{A \widetilde{S_{t}} S h^{2}}\left(\widetilde{u_{f}} \sqrt{A^{2}-{\widetilde{u_{f}}}^{2}}-\sqrt{A^{2}-1}\right)-\ldots \\
& \ldots-A \widetilde{\delta} \frac{S h-\sqrt{A^{2}-1}}{\widetilde{S}_{t} S h^{2}}\left(\arcsin \frac{\widetilde{u_{f}}}{A}-\arcsin \frac{1}{A}\right)
\end{aligned}
$$

Inserting $\widetilde{u_{f}}=\widetilde{u_{f}^{s}}$ from eq.(10) gives the total transient time $\widetilde{t^{s}}$.

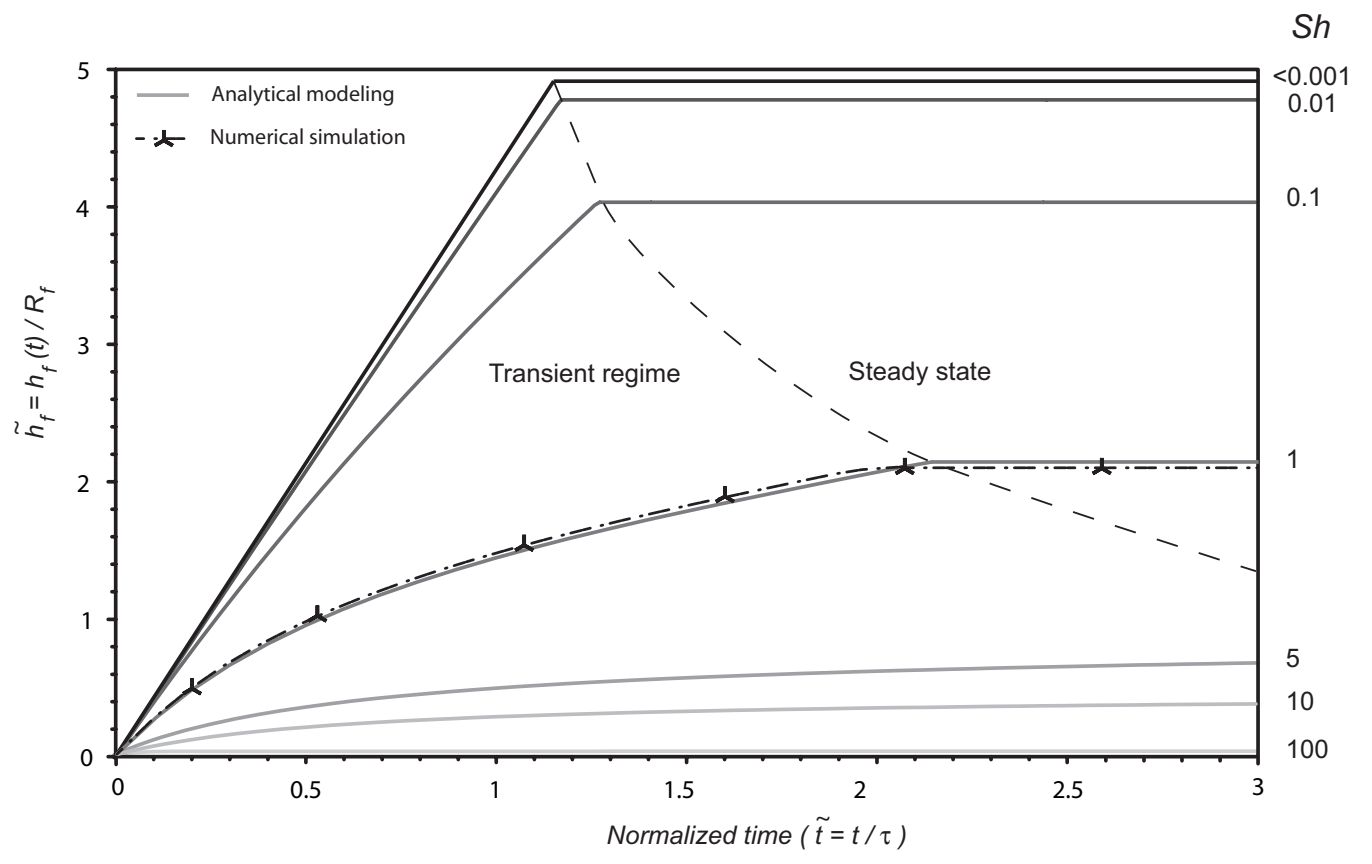

Figure 7: Fiber height versus time $\left(A=5, \widetilde{S}_{t}=2\right)$.

These equations are also useful to assess the effective reaction rate as a function of time, since $\widetilde{k}_{y}^{\text {eff }}$ is a function of $\widetilde{u_{f}}(\widetilde{t})$. Figure 7 is an example of 
solution curves $\widetilde{h_{f}}(\widetilde{t})$ for various values of $S h\left(\right.$ with $A=5, \widetilde{S}_{t}=2, \widetilde{\delta}=2$ ). In the reaction-limited regime, as expected, the growth rate of $\widetilde{h_{f}}$ is proportional to $\tilde{t}$, the proportionality coefficient being $A-1$. The integration method used to plot his figure is presented in Appendix C. The duration of the transient regime in reaction limited-regime $(S h \rightarrow 0)$ is given by the following equation :

$$
t^{s}=\tau \widetilde{t^{s}} \approx \tau \frac{\sqrt{A^{2}-1}}{A-1}
$$

The transient period duration is plotted in figure 8 against $S h$ for several values of $A$. As shown in figures 7 and 8 , the scaled transient regime duration increases with the Sherwood number. This is because, all other variables being fixed, increasing the Sherwood number means decreasing the diffusion coefficient - that is, the oxidant availability. In this model, there is a divergence of the transient time when $S h \rightarrow \sqrt{A^{2}-1}\left(\right.$ i.e. when $\widetilde{u_{f}^{s}} \rightarrow A$ ), which is due to the fact that the reactive flux difference completely vanishes when the final "button" shape is acquired. 


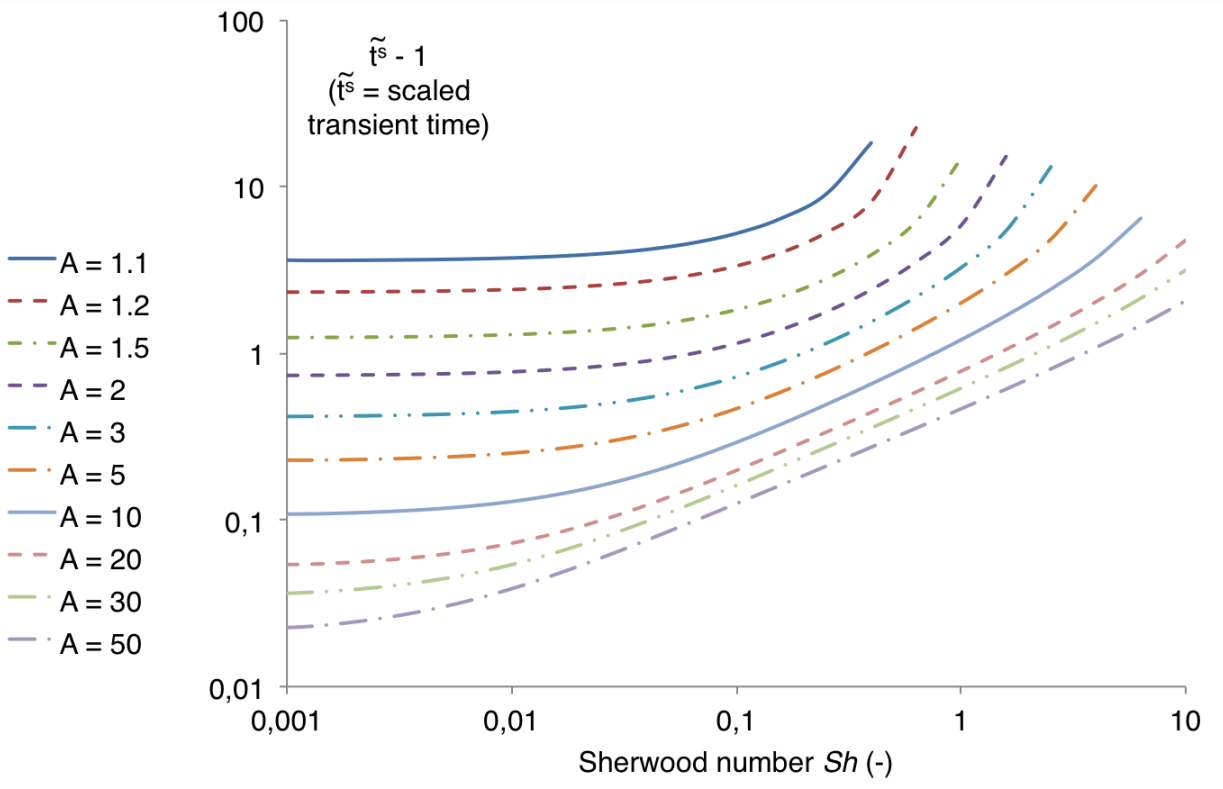

Figure 8: Total transient time duration (in scaled units) vs. the Sherwood number for selected values of the contrast parameter $A$, with $\widetilde{S_{t}}=2$ and $\widetilde{\delta}=2$.

The transient evolution of the fiber shape is represented as a function of time for $S h=1$ in continuous black lines in figure 9. The dashed lines represent the final steady state geometry. On this graph the reference zero ordinate corresponds to the elevation of the weak phase; in other words, it is also the bottom of the uncovered part of the fiber. The transient fiber height plotted in figure 7 is the length measured from bottom of the fiber to its highest elevation. DirectDetailed numerical simulation results used to verify the vertical concentration gradient postulate are plotted on the same graph for convenience, they are explained and discussed in appendix A. 

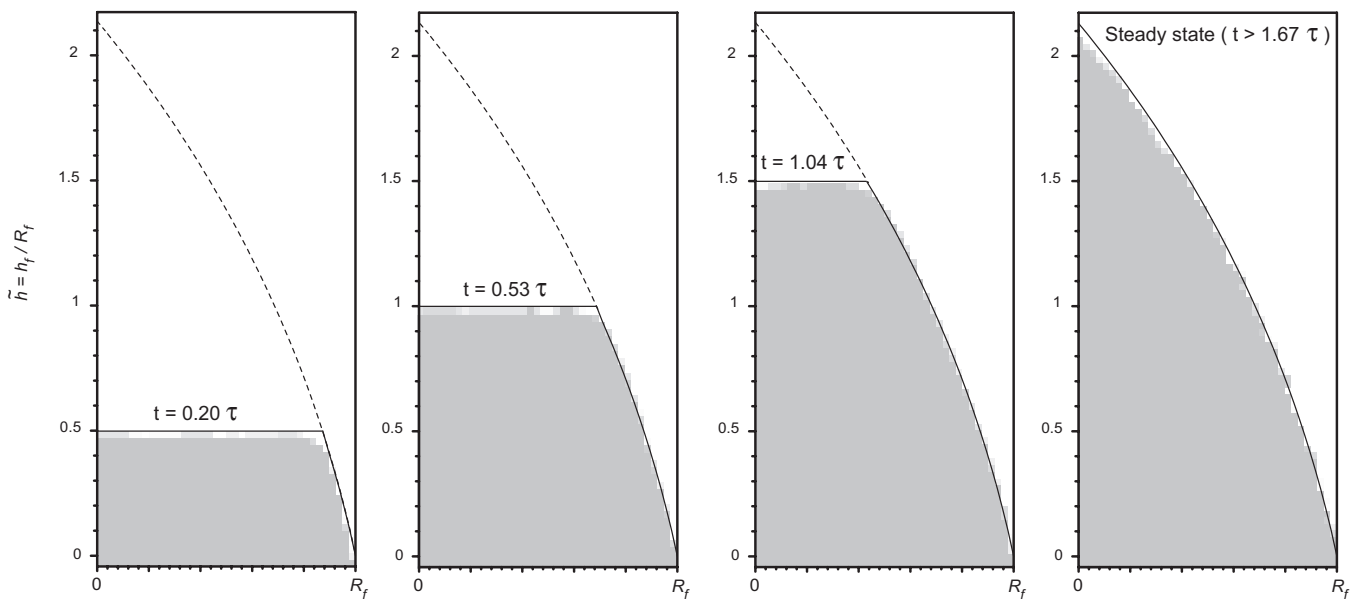

Figure 9: Fiber section morphology versus time : comparison of analytical (black lines) and numerical (grey pixels) results.

\subsection{Macroscopic scale}

The mathematical model of the mesoscopic scale can be applied with little changes to the macroscopic scale to address the second change of scale [4]. Only the material architecture and scales are different as sketched in figures 3 -a and b. The perpendicular yarns $(y)$ are the macroscopic scale equivalents of the fibers. Yarns can be assumed homogeneous, isotropic, strictly perpendicular to the surface, and having a rectangular section surrounded by a weak phase, noted $w p-y$. Between the perpendicular yarns, the composite is alternatively made of parallel yarns $(y-\|)$ and matrix octets $(o)$. In steady state, the composite behavior is only driven by the weakest and the strongest phases, that is, the interphase and the perpendicular yarns [4]. In transient regime, the other phases display little influence on the material behavior if their reactivity is equal to the average reactivity of perpendicular yarns and interphase weighted by their respective surfaces at initial time, 
that is

$$
k_{y-\|}=k_{o}=\frac{k_{y} S_{y}+k_{w p-y} S_{w p-y}}{S_{w p-y}+S_{y}}
$$

For the material of this study, this relation is quite well respected as $S_{y} \simeq$ $6 S_{w p-y}[31], k_{y} \simeq 8 k_{w p-y}[4]$, and $k_{y-\|} \simeq k_{o} \simeq 2 k_{y}[32]$. The macroscopic model of the composite architecture can then be simplified into a model similar to the mesoscopic one, apart from the facts that the perpendicular yarns display a square section (instead of circular for the fibers) and the dimensions are obviously different. The geometry of a yarn is more efficiently described in cartesian coordinates: $S_{y}(x, y, z, t)=z-F(x, y, t)=0$. The surface function $z=F(x, y, t)$ has been obtained in steady state following exactly the same method as for the fibers (but in cartesian coordinates) [4]. The yarns are found to acquire a pyramidal shape and their reduced height is given by :

- if $S h_{y}<\sqrt{A_{y}^{2}-1}$

$$
\widetilde{h_{y}^{s}}=\sqrt{S h_{y}^{-2}+2 S h_{y}^{-1} \sqrt{A_{y}^{2}-1}-1}-S h_{y}^{-1}
$$

- else

$$
\widetilde{h_{y}^{s}}=\left(A_{y}-1\right) / S h_{y}
$$

where $\widetilde{h_{y}^{s}}=2 h_{y}^{s} / l\left(l / 2\right.$, one-half of the yarn edge size - the same way $R_{f}$ was one-half of the fiber diameter), $S h_{y}=k_{w p-y} l /(2 D)$ is the Sherwood number associated to the yarn, $A_{y}=\left(k_{w p-y} \Omega_{w p-y}\right) /\left(k_{y} \Omega_{y}\right)$ the reactivity contrast. Let $\widetilde{\Lambda}=2 \Lambda / l$ be the equivalent of $\widetilde{R}$ in cartesian coordinates; its expression is given by equation (7) after replacing with suitable dimensionless numbers. 
The normalized effective reactivity of the composite as a function of $\widetilde{h_{y}}(t)$ is then

$$
\widetilde{k}_{c}^{e f f}\left(\widetilde{h_{y}}\right)=\frac{\widetilde{\Lambda}^{2}\left(\widetilde{h_{y}}\right)}{A_{y}{\widetilde{S_{t-y}}}_{t}}+\left(1-\frac{\widetilde{\Lambda}^{2}\left(\widetilde{h_{y}}\right)}{{\widetilde{S_{t-y}}}_{t}}\right) \frac{1}{1+S h_{y} \widetilde{h_{y}}}
$$

with $\widetilde{k}_{c}^{e f f}=k_{c}^{e f f} / k_{w p-y}, \widetilde{S}_{t-y}=S_{t-y} /\left(l^{2}\right)$ (where $S_{t-y}$ is the total surface of the cell), and $\widetilde{h_{y}}=2 h_{y}(t) / l$.

Rewriting equation (21) with macroscopic scale variables, one obtains the following differential equation

$$
\frac{d \widetilde{h_{y}}}{d \widetilde{t}}=\frac{1}{1+\widetilde{k}_{c}^{e f f}\left(\widetilde{h_{y}}\right){\widetilde{\delta_{c}^{y}}}^{y} S h_{y}}\left(\frac{A_{y}}{1+\widetilde{h_{y}} S h_{y}}-1\right)
$$

with $\tilde{t}=t / \tau_{y}$ (where $\tau_{y}=l /\left(2 C_{0} k_{y} \Omega_{y}\right)$ is a characteristic time of the macroscopic scale problem), $\widetilde{\delta_{c}^{y}}=2 \delta / l$, and the initial condition for integration being $\widetilde{h_{y}}(t=0)=0$.

The results and discussions of the mesoscopic scale fully apply at the macroscopic scale. For the sake of conciseness, we will not repeat the exact same explanations.

\subsection{Discussion on the validity of the second change of scale}

For the second change of scale, the properties of the yarn are considered constant in space but also in time. This latter assumption is correct if the duration of the transient regime at mesoscopic scale is small compared to that of the macroscopic scale. Let the validity of this hypothesis be evaluated in the case of the $\mathrm{C} / \mathrm{C}$ composite of the study. The $3 \mathrm{D} \mathrm{C} / \mathrm{C}$ material of this study has been oxidized at $898 K$ under dry air and atmospheric pressure. In these conditions, the oxidation rate of the carbon fibers and of the perpendicular yarns are respectively about $1.210^{-5} \mathrm{~m} . \mathrm{s}^{-1}$ [30] and $3.810^{-4} \mathrm{~m} . \mathrm{s}^{-1}$ [4]. 
The contrast in the reactivities are $A=32, A_{y}=8$ [4]. In these conditions, the regime is reaction-limited at both scales. According to equation (25), the theoretical durations of the transient regime, as estimated by the analytical model, are respectively 5 hours at the mesoscopic scale and 26 hours at the macroscopic scale. Therefore, considering that the mesoscopic time scale is small compared to the macroscopic time scale is not fully exact but acceptable.

\section{Validation and analysis}

In a previous work, oxidation experiments were carried out in a laboratory oven under dry air at $898 \mathrm{~K}$ [30]. We will compare observed and predicted surface roughness (subsection 4.1) and mass loss (subsection 4.2) versus time.

\subsection{Surface roughness}

As described in the modeling section, typical surface roughness patterns develop both at the fiber scale and at the yarn scale. Due to the difference in dimensions and time scales, the mesocsopic and the macroscopic scale are separately studied in the following subsections.

\subsubsection{Mesoscopic scale}

SEM micrographs of the typical surface roughness pattern appearing on a perpendicular bundle of the $3 \mathrm{D} \mathrm{C} / \mathrm{C}$ composite of the study are presented in figure 10. The evolution is in full qualitative agreement with the findings of the theoretical analytical study. A more reactive interphase is oxidized at a faster ablation rate. The bulk matrix is progressively ablated from the sides. Protruding fibers take the typical needle shape. A fully conical (pointed) 


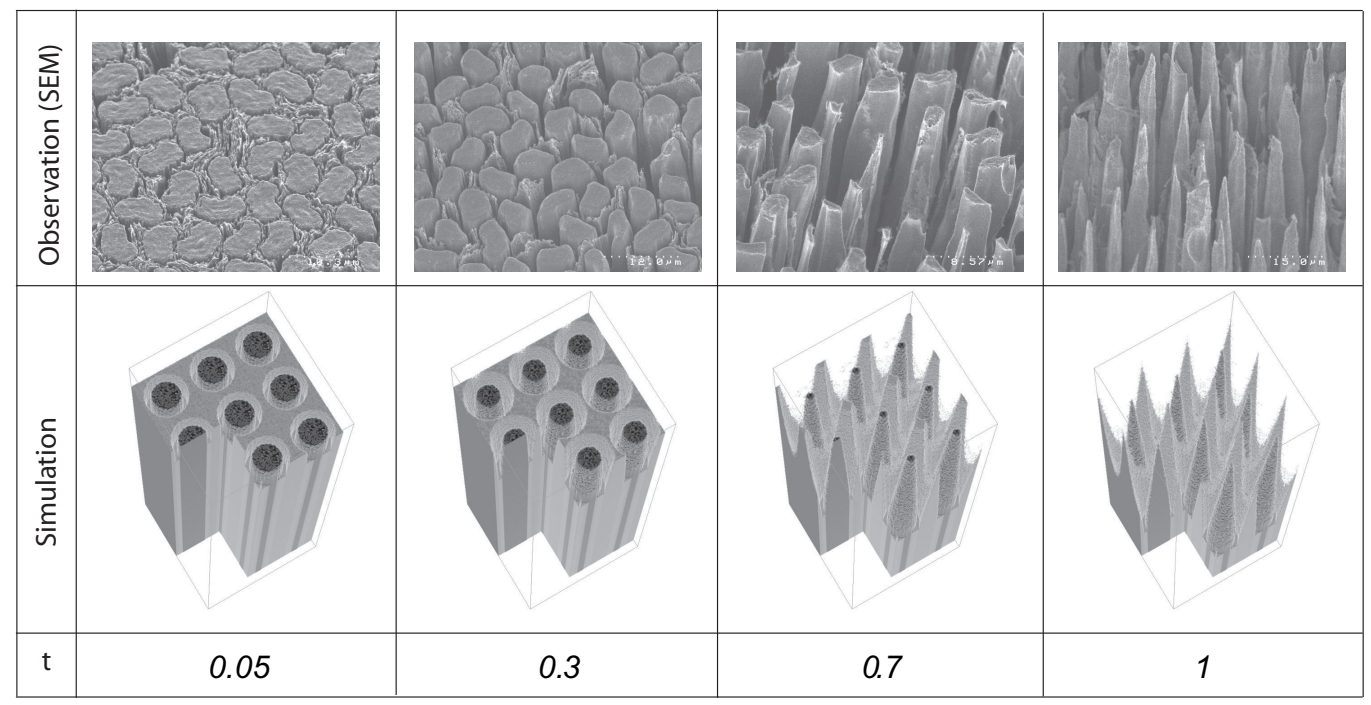

Figure 10: Experimental observations (SEM) vs. simulation results (AMA) as a function of $h_{f} / h_{f}^{s}(A=30, S h<0.001)$.

shape is in agreement with the reaction-limited regime of the experiment [4]. In figure 10, we present directdetailed numerical simulations obtained with the procedure and simulation tool described in appendix A. For the simulations, the reactivities of the composite and of its components have been independently assessed either by direct experimental measurements (composites, fibers) [30] or by inverse analysis (matrices, yarns) [4]. The reactivity contrasts was found to be $A=32$ at the fiber scale and $A_{y}=8$ at the bundle scale. The reference intrinsic reactivity of carbon fibers was independently measured to be $1.2 \cdot 10^{-5} \mathrm{~m} . \mathrm{s}^{-1}$ in the conditions of the experiment [30]. This data set was used for the transient simulations. Zoomed-in SEM micrographs, numerical simulation, and analytical computation are shown in figure 11 during transient regime and in steady state. The results of the 


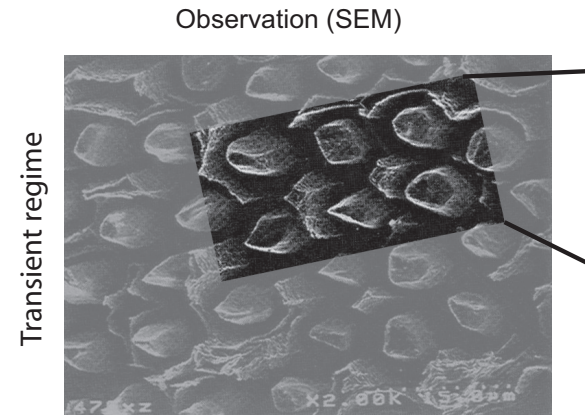

(a)

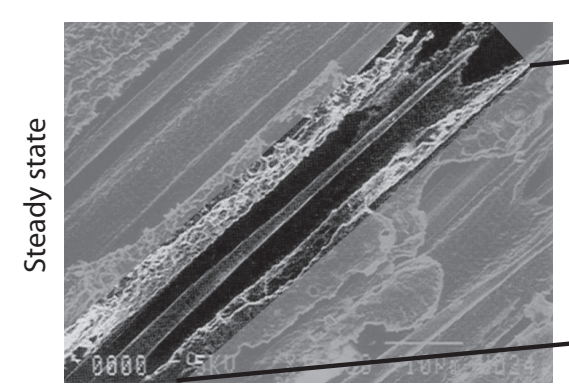

(d)
Simulation (AMA)

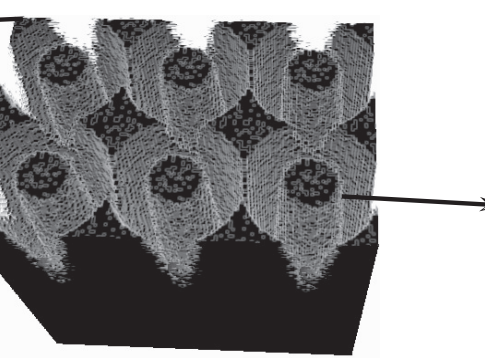

(b)

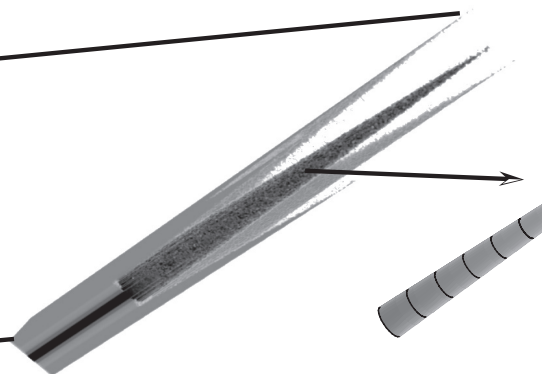

(e)
Analytic (computed)

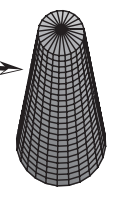

(c)

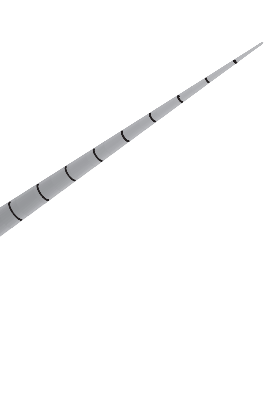

(f)

Figure 11: Comparison of experimental and theoreticalmodel results in transient regime and in steady state

numerical and analytical models are in fullqualitative agreement with experimental observations. Quantitative agreement could not be checked by lack of reliable experimental measurements of fiber heights.

\subsubsection{Macroscopic scale}

Macroscopic scale micrographs taken after the end of the oxidation test are shown in figure 12-a. The yarns display truncated pyramidal shapes. This morphology strongly suggests that steady state was not reached at the end of the oxidation test. Sharp pyramidal shapes would be expected form the theoretical models. In section 3.3, the theoretical duration of the transient 
regime was estimated from the models to be 26 hours, while the reference experiment lasted 17 hours [30]. In reaction-limited regime the yarn growth is a linear function of time. With $A_{y}=8$, equation (27) provides a value of $h_{y}^{s}$ of four times the yarn edge size $(l)$. Hence, the truncated fiber height of figures $12-\mathrm{b}$ and $\mathrm{c}$ is predicted to be around $2.5 \mathrm{l}$. This is in full agreement with the experimental yarn height measured from micrographs 12-b and c. Simulations have been carried out on the numerical cell presented in figure 12-b, and designed according to micrograph 12-a. The different phase properties are taken from the results of the validated stead-state study [4]. All the component effective densities are equal to $2 \mathrm{~g} . \mathrm{cm}^{-3}$, except for the matrix octets which are not as dense $\left(1.6 \mathrm{~g} . \mathrm{cm}^{-3}\right)$. Apart from the interphase that is height times more reactive $\left(A_{y}=8\right)$, all phases have the same intrinsic reactivity. The reactivity of the bundles is obtained from the mesoscopic scale upscaling, and found to be $3.8 \cdot 10^{-4} \mathrm{~m} \cdot \mathrm{s}^{-1}$ [4]. The theoretical results for an oxidation duration of 17 hours are shown in figures 12-e (directdetailed numerical simulation) and 12-f (analytical surface function). Theoretical results are in good agreement, qualitatively and quantitatively, with the experimental observations. This analysis confirms that the transient regime had not been fully reached after 17 hours of oxidation, to the contrary of what the authors of the experimental study may have observed when following mass loss only [30]. This is further investigated with detailed mass loss analysis in the following subsection.

\subsection{Mass loss in transient regime}

In the experimental study, the mass loss has been experimentally measured versus time from the initial time to the end of the experiment (17 


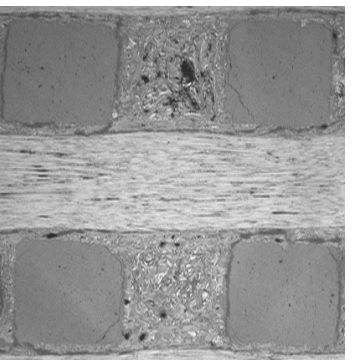

a) Initial time (SEM)

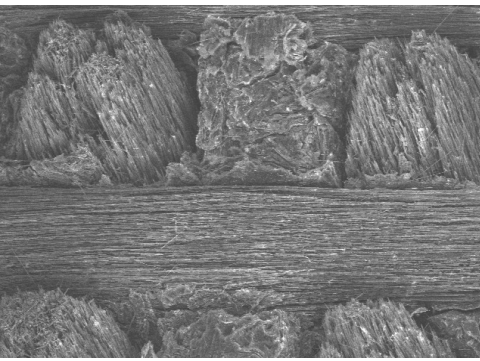

b) End of the experiment (SEM) : transient regime

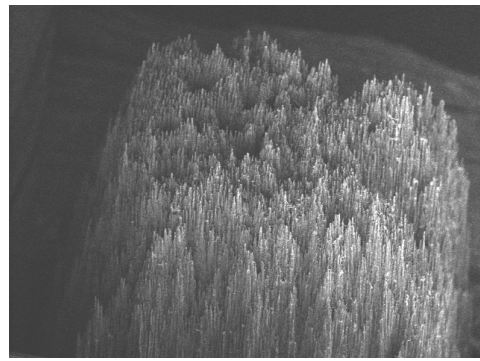

c) Zoom on a fiber bundle (SEM)

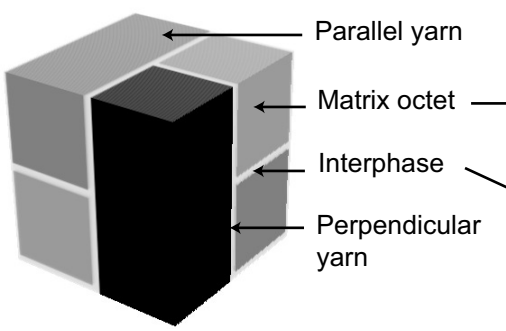

d) Initial time : Numerical cell (AMA)

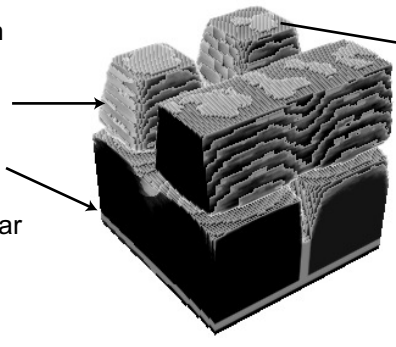

e) Transient regime : Simulation (AMA)

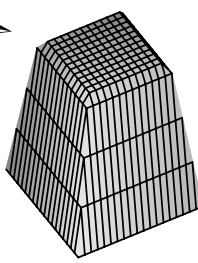

f) Transient regime : Analytical computation

Figure 12: Comparison of experimental and theoretical morphologies at macroscopic scale

hours). The mass loss at 17 hours is provided in gram per minute in [30] for a sample of one square centimeter surface. On figure 13, the experimental mass loss is provided in dimensionless Figure 13 shows the experimental mass loss in nondimensional units $(\widetilde{m})$ as a function of the reduced time $\left(\widetilde{t}=t / \tau_{y}\right)$. The duration of the transient regime at mesoscopic scale is small compared to the duration of the transient regime at macroscopic scale. We will then neglect the short mescoscopic scale transient and evaluate the transient mass loss of the composite directly from its macroscopic behavior. The total mass loss at time $t$ is given by

$$
m(t)=\mathcal{M}_{c} S_{e f f} \int_{0}^{t} k_{c}^{e f f}(v) C_{e f f}(v) d v
$$


where $S_{\text {eff }}$ is the effective geometric surface of the sample and $\mathcal{M}_{c}$ the molar mass of carbon. In this experiment, it has been shown that $C_{\text {eff }}=C_{0}[30]$. Using this hypothesis, let equation (31) be rewritten under a dimensionless form

$$
\widetilde{m}(\widetilde{t})=\int_{0}^{\widetilde{t}} \widetilde{k}_{c}^{e f f}(v) d v
$$

with

$$
\widetilde{m}(\widetilde{t})=m(\widetilde{t}) \frac{k_{y} \Omega_{y}}{k_{w p-y} \mathcal{M}_{c} S_{e f f} l}
$$

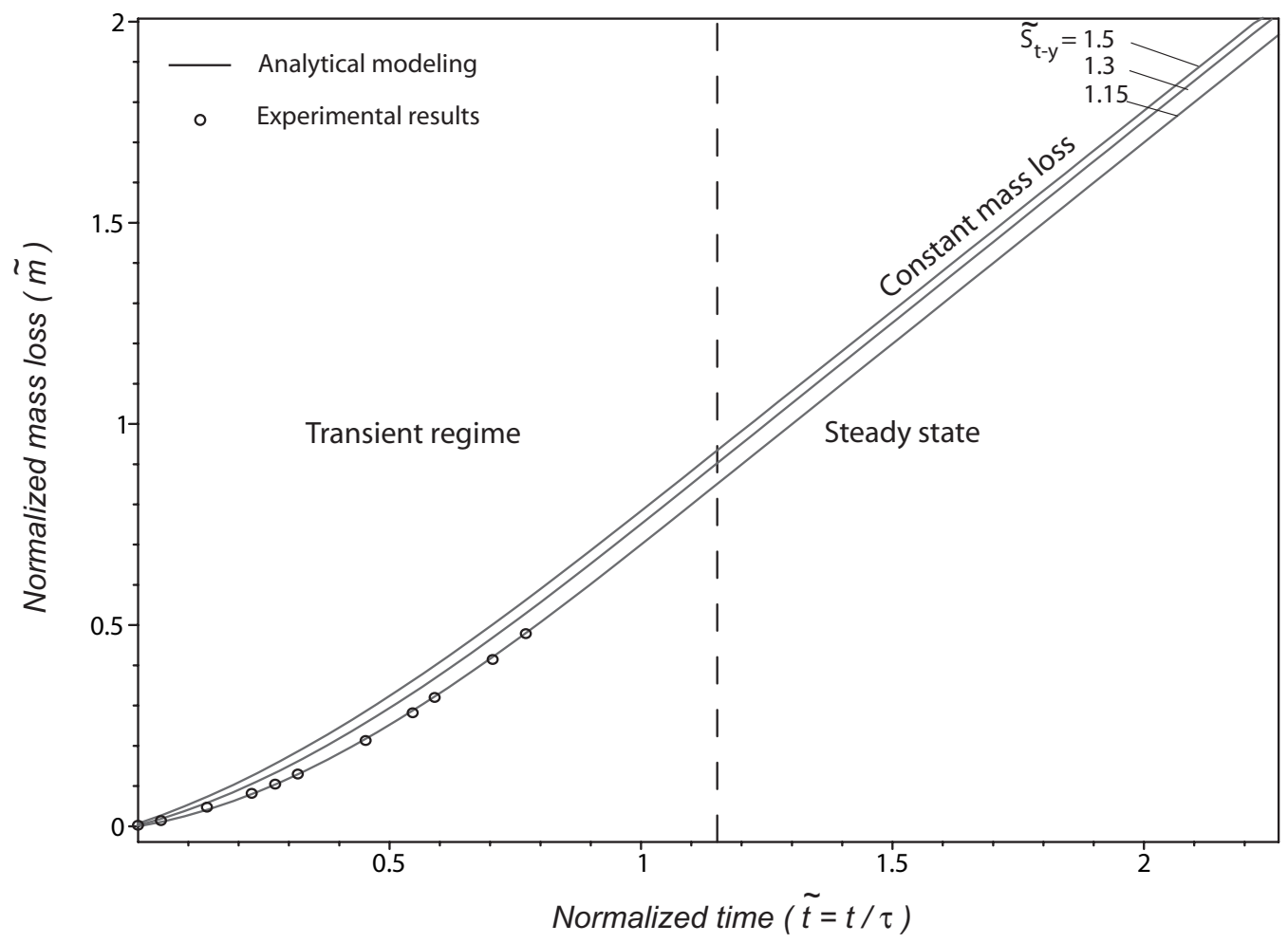

Figure 13: Comparison of experimental and theoretical mass loss (3DCC, dry air oxidation, $898 K)$ 
In order to plot the theoretical mass loss, the proportion of inter-yarn interphase in the eall hole has to be estimated. According to micrograph 12-a, it lies around $15 \%$. The value of $\widetilde{S}_{t-y}$ is then about 1.15 . The steady state mass loss is not a function of $\widetilde{S}_{t-y}$. However, at initial time, the proportion of weak phase has a neat marked influence on the mass loss rate as shown in figure 13, for different values of $\widetilde{S}_{t-y}$, namely $1.15,1.3$, and 1.5. Using the measured value of 1.15 , the agreement between experimental and theoretical results is excellent. At the end of the experiment (17 hours, or in dimensionless time $\widetilde{t}=0.75$ ), the mass loss rate appeared to be constant and the experiment has been stopped. The fact that the oxidation rate seems constant before the end of the transient regime is explained by the almost linear trend of predicted mass loss at the end off the transient phase; it is plotted in figure 13. This is in agreement with the theoretical results plotted in figure 5: the effective reactivity tends to a horizontal asymptote at the end of the transient regime. According to equation (29), at the end of the 17 hour experiment the mass loss rate was only $90 \%$ of the theoretical mass loss rate in steady state. This theoretical results bring the opportunity to re-examine the previously published results. First, the steady state reactivity of the 3D $\mathrm{C} / \mathrm{C}$ is about $10 \%$ higher than measured, that is, its actual geometrical reactivity is $3.310^{-3} \mathrm{~m} \cdot \mathrm{s}^{-1}$ at $898 \mathrm{~K}$, under dry air and atmospheric pressure [30].

As a conclusion for the validation section, it can be said that typical surface roughness features are well captured and explained by the model and that a correct quantitative agreement is obtained on the mass loss prediction. 


\section{Conclusion}

A validated steady state model has been extended to capture the transient regime behavior of a $3 \mathrm{D} \mathrm{C} / \mathrm{C}$ composite. A reaction/diffusion model has been proposed and solved analytically. First, the mesoscopic scale behavior of the composite (perpendicular bundle) is inferred from the properties of the microscopic scale components (fiber, intra-yarn matrix). Second, the macroscopic scale behavior of the composite is obtained from that of the bundle and the inter-bundle components. DirectDetailed numerical simulation are presented in an appendix to validate two postulates used in the analytical model (vertical mass transfer, representativeity of the perpendicular fiber and yarn model for other orientations). The model provides comprehensive understanding of the ablative behavior of carbon composites under mild oxidation conditions: evolution of surface roughness as a function of time, effective reactivity of the composite, and overall mass loss. Finally, the model was validated by comparison to an oxidation experiment carried out on a $3 \mathrm{D} \mathrm{C} / \mathrm{C}$ composite in reaction-limited regime, with excellent prediction of the surface roughness features and of the mass loss rate. An outlook of this work is to apply and verify the validity of this approach to other oxidation conditions for which the reaction regime would be partially diffusion limited (higher reactivity), and to sublimation. Obviously, due to the thinning of perpendicular fibers and loss of cohesion of parallel fibers, mechanical erosion (spallation) models should be included in the model for high shear-stress conditions. 


\section{Acknowledgments}

The authors wish to thank J.-F. Epherre, J.-M. Goyhénèche and G. Duffa (CEA) for fruitful discussions. The authors also wish to thank CNRS, CEA, and SPS for financial support.

[1] G. Savage, Carbon/Carbon composites, Chapman \& Hall, London, 1993.

[2] E. Fitzer, L. M. Manocha, Carbon reinforcements and C/C composites, Springer, 1998, $342 \mathrm{p}$.

[3] G. Duffa, Ablative Thermal Protection Systems Modeling, AIAA Education Series, 2013. doi:10.2514/4.101717.

[4] J. Lachaud, Y. Aspa, G. L. Vignoles, Analytical modeling of the steady state ablation of a 3D $\mathrm{C} / \mathrm{C}$ composite, International Journal of Heat and Mass Transfer 51 (9-10) (2008) 2614-2627, doi:10.1016/j.ijheatmasstransfer.2008.01.008.

[5] X. Bourrat, Sciences of carbon materials, Universidad de Alicante, Alicante, 2000, Ch. 1. Structure in carbons and carbon artifacts, pp. 1-97.

[6] A. K. Geim, K. S. Novoselov, The rise of graphene, Nature Materials 6 (2007) 183-191. doi:10.1038/nmat1849.

[7] J. M. Thomas, Microscopic studies of graphite oxidation, Chemistry and Physics of Carbon 1 (1965) 121-202.

[8] L. Bonnetain, G. Hoynant, Oxydation des graphites aux tempï $; \frac{1}{2}$ ratures moyennes, Vol. 1 of Les Carbones, Masson, Paris, 1965, Ch. 17, pp. 277382. 
[9] N. R. Laine, F. J. Vastola, P. R. Walker, The importance of active surface area in the carbon-oxygen reaction, J. Phys. Chem. 67 (1963) 2030-2034.

[10] P. Ehrburger, J. Lahaye, Characterization of carbon-carbon composites: oxiation behavior, Carbon 19 (1981) 7-10.

[11] S. Goto, K. H. Han, G. R. St-Pierre, A review on oxidation kinetics of carbon fiber/carbon matrix composites at high temperature, Trans. Iron Steel Inst. Jpn. 26 (1986) 597-603.

[12] K. Kuo, S. Keswani, A comprehensive theoretical model for carboncarbon composite nozzle recession, Combustion Science and Technology 42 (1986) 177-192.

[13] D. W. McKee, Oxidation behavior and protection of carbon/carbon composites, Carbon 25 (4) (1987) 551-557.

[14] K. L. Luthra, Oxidation of C/C composites - A theoretical study, Carbon 26 (2) (1988) 217-224.

[15] J. Lahaye, F. Louys, P. Ehrburger, The reactivity of carbon-carbon composites, Carbon 28 (1) (1990) 137-141.

[16] D. Cho, J. Y. Lee, B. I. Yoon, Microscopic observations of the ablation behaviours of carbon fibre/phenolic composites, Journal of Materials Science Letters 12 (1993) 1894-1896.

[17] W. H. Glime, J. D. Cawley, A. J. Eckel, The evolution of a non-planar ox- 
idation front in receding carbon fibers, Ceramic Transactions 46 (1995) 1039-1047.

[18] J. Rodriguez-Mirasol, P. A. Thrower, L. R. Radovic, On the oxidation resistance of carbon-carbon composites: importance of fiber structure for composite reactivity, Carbon 33 (4) (1995) 545-554.

[19] J. C. Han, X. D. He, S. Y. Du, Oxidation and ablation of 3D carboncarbon composite at up to 3000 C, Carbon 33 (4) (1995) 473-478.

[20] S. Labruquere, X. Bourrat, R. Pailler, R. Naslain, Structure and oxidation of C/C composites: role of the interface, Carbon 39 (2001) 971-984.

[21] M.-P. Bacos, J.-M. Dorvaux, O. Lavigne, Y. Renollet, C/C composite oxidation model : I. Morphological experimental investigations, Carbon 38 (2000) 77-92.

[22] M.-P. Bacos, J.-L. Cochon, J.-M. Dorvaux, O. Lavigne, C/C composite oxidation model : II. Oxidation experimental investigations, Carbon 38 (2000) 93-103.

[23] M.-P. Bacos, J.-M. Dorvaux, O. Lavigne, J. Talandier, C/C composite oxidation model :III. Physical basis, limitations and applications, Carbon 38 (2000) 105-117.

[24] D. Cho, B. I. Yoon, Microstructural interpretation of the effect of various matrices on the ablation properties of carbon-fiber-reinforced composites, Composites science and technology 61 (2001) 271-280. 
[25] R. Luo, J. Cheng, T. Wang, Oxidation behaviour and protection of carbon/carbon composites prepared using rapid directional diffused CVI techniques, Carbon 40 (2002) 1965-1972.

[26] Y.-J. Lee, H. J. Joo, Investigation on ablation behavior of CFRC composites prepared at different pressures, Composites: Part A 35 (2004) $1285-1290$.

[27] J. Lachaud, G. L. Vignoles, J. M. Goyheneche, J. F. Epherre, Ablation in $\mathrm{C} / \mathrm{C}$ composites: microscopic observations and 3D numerical simulation of surface roughness evolution, Ceramic Transactions 191 (2006) 149160.

[28] W. Guo, H. Xiao, E. Yasuada, Y. Cheng, Oxidation kinetics and mechanisms of a 2D-C/C composite, Carbon 44 (2006) 3269-3276.

[29] N. S. Jacobson, D. M. Curry, Oxidation microstructure studies of reinforced carbon/carbon, Carbon 44 (2006) 1142-1150.

[30] J. Lachaud, N. Bertrand, G. L. Vignoles, G. Bourget, F. Rebillat, P. Weisbecker, A theoretical/experimental approach to the intrinsic oxidation reactivities of $\mathrm{C} / \mathrm{C}$ composites and of their components, Carbon 45 (14) (2007) 2768-2776, doi:10.1016/j.carbon.2007.09.034.

[31] G. Vignoles, J. Lachaud, Y. Aspa, J. M. Goyhénèche, Ablation of carbon-based materials: multiscale roughness modeling, Composites Science and Technology 69 (9) (2008) 1470-1477, doi:10.1016/j.compscitech.2008.09.019. 
[32] J. Lachaud, G. L. Vignoles, A brownian motion technique to simulate gasification and its application to $\mathrm{c} / \mathrm{c}$ composite ablation, Computational Material Science 44 (4) (2008) 1034-1041, doi:10.1016/j.commatsci.2008.07.015.

[33] G. Vignoles, J. Lachaud, Y. Aspa, M. Quintard, Effective surface recession laws for the physico-chemical ablation of $\mathrm{C} / \mathrm{C}$ composite materials, in: S. Mathur, T. Ohji (Eds.), Mechanical Properties and Performance of Engineering Ceramics and Composites, Dileep Singh and Jonathan Salem, V. Ceram. Eng. And Sci. Procs (31) 2, The American Ceramic Society, Westerville (OH), 2010, pp. 351-360.

[34] G. Vignoles, J. Lachaud, Y. Aspa, Environmental effects: Ablation of c/c materials - surface dynamics and effective reactivity, in: N. P. Bansal, J. Lamon (Eds.), Ceramic Matrix Composites: Materials, Modeling and Technology., John Wiley and Sons, Inc., Hoboken, NJ, 2014.

[35] G. Vignoles, Y. Aspa, M. Quintard, Modelling of carbon-carbon composite ablation in rocket nozzles, Composites Science and Technology 70 (9) (2010) 1303-1311, doi:10.1016/j.compscitech.2010.04.002.

[36] S. Pestchanyi, V. Safronov, I. Landman, Estimation of carbon fibre composites as ITER divertor armour, Journal of Nuclear Materials 329-333 (2004) 697-701.

[37] J. Lachaud, J. B. Scoggins, T. E. Magin, M. G. Meyer, N. N. Mansour, A generic local thermal equilibrium model for porous reactive materials submitted to high temperatures, Interna- 
tional Journal of Heat and Mass Transfer 108 (2017) 1406-1417. doi:10.1016/j.ijheatmasstransfer.2016.11.067.

[38] J. Lachaud, I. Cozmuta, N. N. Mansour, Multiscale approach to ablation modeling of phenolic impregnated carbon ablators, Journal of Spacecraft and Rockets 47 (6) (2010) 910-921, doi: 10.2514/1.42681.

[39] I. V. Katardjiev, G. Carter, M. J. Nobes, S. Berg, H.-O. Blom, Threedimensional simulation of surface evolution during growth and erosion, Journal of Vacuum Science and Technology A 12 (1) (1994) 61-68.

[40] J. C. Ferguson, F. Panerai, J. Lachaud, A. Martin, S. C. Bailey, N. N. Mansour, Modeling the oxidation of low-density carbon fiber material based on micro-tomography, Carbon 96 (2016) 57-65, doi:10.1016/j.carbon.2015.08.113.

[41] P. Schrooyen, K. Hillewaert, T. E. Magin, P. Chatelain, Fully implicit discontinuous galerkin solver to study surface and volume ablation competition in atmospheric entry flows, International Journal of Heat and Mass Transfer 103 (2016) 108-124. doi:10.1016/j.ijheatmasstransfer.2016.07.022. 


\section{Appendix A. Verification of the postulates}

The analytical solution has been obtained under two postulates: (i) the gradient of reactant concentration is only vertical (along $\mathbf{z}$ ), (ii) the effective reactivity of the elemental cell (fiber and weak phase) is not a function of the fiber axis orientation.

The objective of this appendix is to verify these postulates by directdetailed numerical simulation. First, the numerical simulation tool is presented (subsection 1). Then, the mathematical model is numerically solved in the case of a perpendicular fiber to verify the vertical concentration gradient hypothesis (subsection 2). Finally, the case of non perpendicular orientations is studied (subsection 3).

Presentation of $A M A$

An efficient numerical simulation code, named AMA, has been developed on a Monte-Carlo random-walk principle to solve the model presented in section 2.2 [32]. AMA, which has been implemented in ANSI C, contains five main parts:

- (i) A 3-D image containing several phases (fluid/solids) is described by discrete cubic voxels (3D pixel).

- (ii) The moving fluid/solid interface is determined by a simplified marching cube approach.

- (iii) Mass transfer by diffusion is simulated by a Brownian motion simulation technique, which is a continuum (grid-free) method to simulate diffusion. 
- (iv) Heterogeneous first-order reaction on the wall is simulated by a sticking probability adapted to the Brownian motion simulation technique.

- $(v)$ A Dirichlet upper boundary condition is simulated using a buffer zone, where $C$ is maintained constant at $C_{0}$.

AMA has been validated by comparison to a 1D analytical model in transient regime and to the $3 \mathrm{D}$ mesoscopic scale model in steady state. Independent implementations of the same model have been successfully compared to AMA $[35,40,41]$.

Verification of the vertical concentration gradient hypothesis

The transient regime has been simulated on the cell presented in subsection 3.1 for $A=5$. The steady state is reached after a computing time of 48 hours on $3.2 \mathrm{GHz}$ single core. The difference between numerical and analytical results is found to be lower than $3 \%$ for both estimations of the duration of the transient regime and fiber height as a function or time, in all regimes. We propose an illustration for the most complex case, that is, in mixed regime for $S h=1$. Analytical and numerical fiber morphologies are compared in figure 9. The error is equal to the voxel size, that is the maximal accuracy of the numerical method. The numerical prediction of the roughness growth as a function of time is reported in figure 7. The steady state height is $2.5 \%$ lower for the numerical simulation than for the analytical evaluation (corresponding to the pixel approximation). Consequently, the vertical concentration gradient hypothesis is acceptable, as directdetailed numerical simulation results and analytical results obtained under the vertical 
gradient hypothesis match each other within less than $3 \%$.

Numerical analysis of non perpendicular orientations : effect of obliquity and parallelism

The analytical model has been developed for perpendicular fiber orientations. Clearly, such perfect orientations are observed only in rare occasions. There are two other cases that need to be studied. First, we will study the case of fibers belonging to perpendicular bundles and displaying an oblique orientation with the effective surface bundle surface. Second, we will study the case of fibers belonging to parallel bundles and displaying a parallel orientation with the bundle's effective surface. In both cases, we will show by directdetailed numerical simulation that the perpendicular orientation results hold for all orientations.

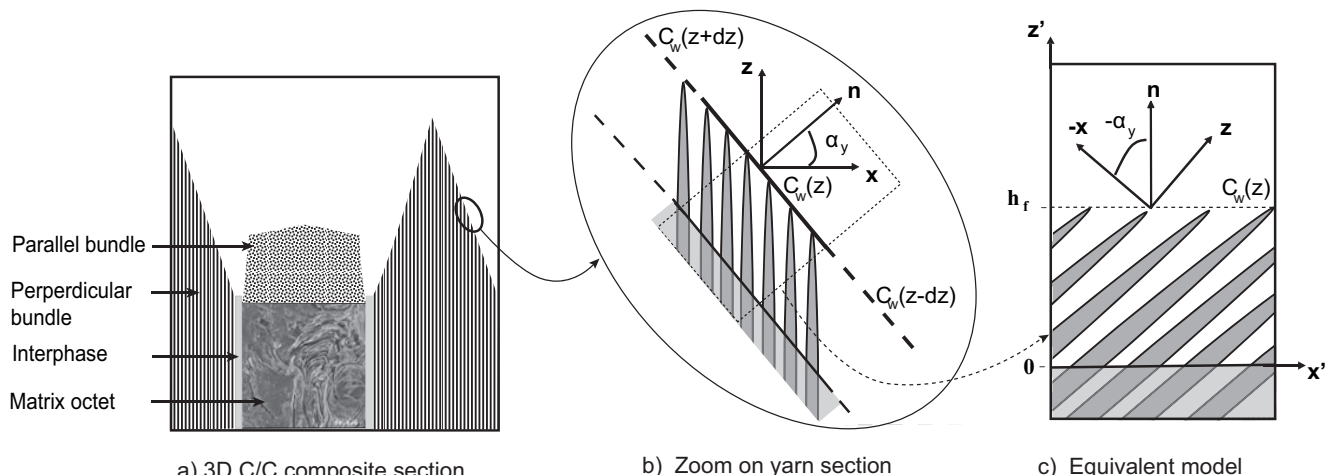

Figure A.14: Scheme of the multiscale surface roughness on a 3D C/C in steady state

As represented in figure A.14, when the perpendicular yarns take their typical pyramidal shape, the local surface normal $\mathbf{n}$ features an angle $\alpha_{y}$ with the horizontal axis. The effective yarn surface is defined by the tips 


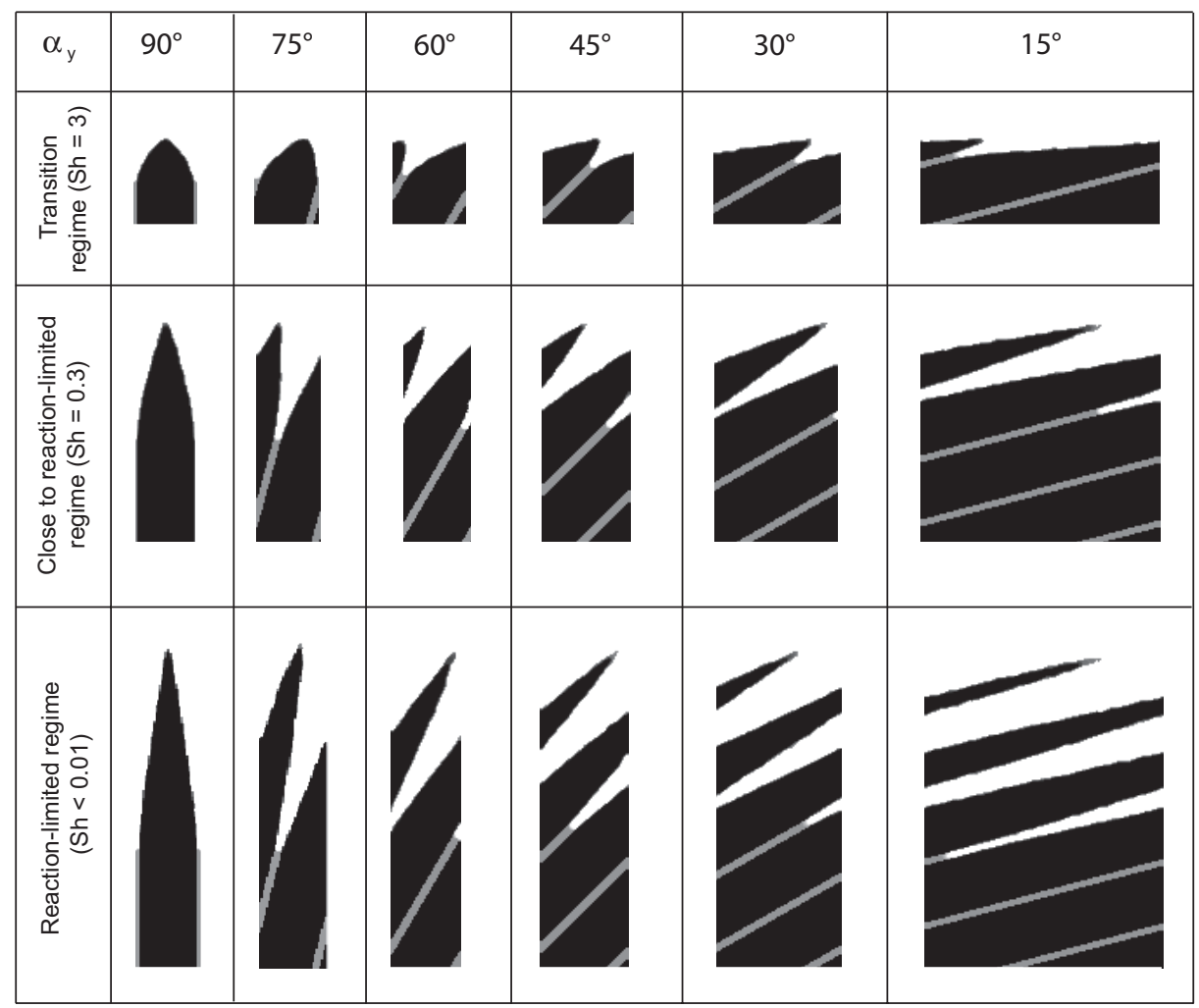

Figure A.15: Comparison of the surface roughness features when $\alpha_{y}$ varies $(A=10)$.

of the fibers and the local surface recession occurs along $-\mathbf{n}$. The local 3-D cell can be represented as shown in figure A.14-c, where $C_{w}(z)$ is the local concentration on the bundle's surface. This configuration has been analyzed by directdetailed numerical simulation in three dimensions for fiber orientations ranging from $90^{\circ}$ to $15^{\circ}$, by steps of $15^{\circ}$. Steady state results are presented in figure A.15 for $A=10$ and for various regimes; only the diffusion-limited regime is not represented because it is trivial (flat surface). In reaction-limited regime $(S h<0.01)$, the fiber height $h_{f}^{s}$, expressed as the 
vertical distance from the weak phase to the fiber tip, is not a function of $\alpha_{y}$. Tortuosity starts playing a role when diffusional effects appear $(S h>0.01)$ and $h_{f}^{s}$ is found to slightly increase with $\alpha_{y}$ as seen in figure A.15. Indeed, the diffusion path of the oxidant through oblique fibers is more tortuous, and the weak phase is better protected by oblique fibers. However, in all regimes, effective reactivity variations with fiber tilt angle are found to be below the simulation code uncertainty $(<2.5 \%)$. Therefore, the bundle effective reactivity can be considered homogeneous. This confirms the validity of the second postulate for perpendicular yarns.

In parallel yarns, fibers are parallel to the surface. As shown in figure A.16, carbon fibers are surrounded by a weak phase of ex-pitch carbon matrix, called interphase. Its thickness is about $0.5 \mu \mathrm{m}$. The rest of the inter-fiber space is filled by a bulk pitch-based matrix whose reactivity is equal to that of the fibers.

In the oxidation laboratory experiment, no erosion was observed. The fibers are very long and not rigorously straight. SEM observations show that they keep some degree of linkage until they are fully ablated [4]. It is however well known that this material is subject to spallation in high shear-stress flows [3]. Mechanical erosion was neglected in the simulations presented in figure A.16 obtained from reaction-limited regime $(S h<<1)$ to diffusion-limited regime $(S h>>1)$ for $A=30$. The value of the normalized effective reactivity $\left(\widetilde{k}=k_{e f f} / k_{w p}\right)$ decreases from around unity in reaction-limited regime to close to $1 / A$ in reaction limited regime. These values are very close to those obtained with the perpendicular fiber analytical model. The maximal discrepancy is obtained in diffusion limited regime $(\sim 17 \%)$. Nevertheless, 


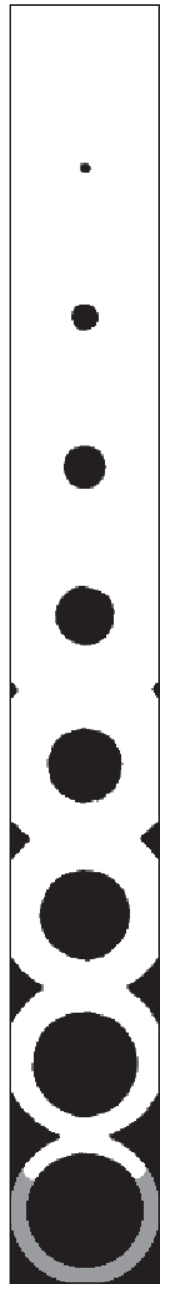

a) $\underset{\widetilde{k} \sim 1}{S h}=0.014$

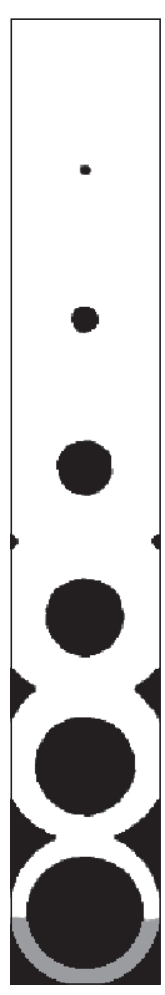

b) $\underset{\widetilde{k}}{\sin }=0.14$

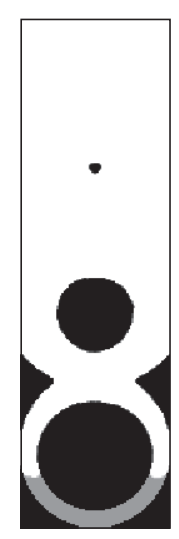

c) $\underset{\widetilde{\mathrm{k}}}{\mathrm{Sh}} \sim 0.13$

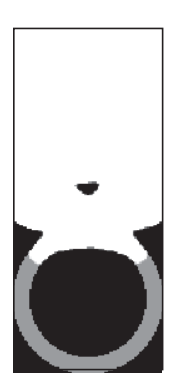

d) $\underset{\sim}{\mathrm{K}}=14$

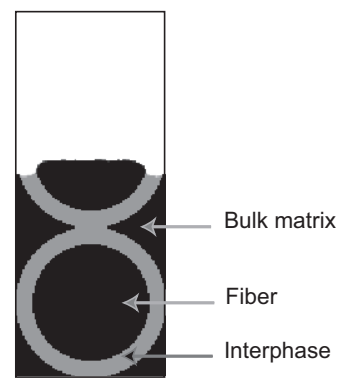

e) $\underset{\widetilde{\mathrm{k}}}{\mathrm{Sh}}=140.04$

Figure A.16: Simulation of a parallel yarn behavior as a function of $S h$ for $A=30$.

as far as the gasification rate is concerned, the error is lower since diffusional effects tends to smooth out the reactivity differences. Then, as a first approximation, the effective reactivity of parallel yarns can be estimated using the perpendicular yarn model. It is clear from the simulations that this is 
only valid when the environmental conditions are such that no mechanical erosion occurs.

\section{Appendix B. Derivation of equation (24)}

Let us use:

- $h$ as a short notation for $\tilde{h_{f}}$

- $t$ as a short notation for $\tilde{t}$

- $k$ as a short notation for $\tilde{k}_{e f f}$

The evolution equation is:

$$
\frac{d h}{d t}=\frac{1}{1+k \delta S h}\left(\frac{A}{1+h S h}-1\right)
$$

where :

$$
k=\frac{1}{1+h S h}+\frac{R^{2}(h)}{S_{t}}\left(\frac{1}{A}-\frac{1}{1+h S h}\right)
$$

in which

$$
R(h)=S h^{-1}\left(\sqrt{A^{2}-(1+h S h)^{2}}-\sqrt{A^{2}-1}\right)+1 \text { for } h \in\left[0 ; \frac{A-1}{S h}\right]
$$

The transient time is given by integration of eq.(B.1), as :

$$
t_{\text {trans }}=\int_{0}^{h^{s}}(1+k \delta S h) \cdot \frac{1+h S h}{A-(1+h S h)} d h
$$

where the steady-state height is :

$$
h^{s}=S h^{-1}\left(\sqrt{1+2 S h \sqrt{A^{2}-1}-S h^{2}}-1\right)
$$


Let us define $u=1+h S h$, so that $d h=S h^{-1} d u$.

Eqs(B.1-B.5) become :

$$
\begin{gathered}
\frac{d u}{d t}=\frac{S h}{1+k \delta S h}\left(\frac{A}{u}-1\right) \\
k=\frac{1}{u}+\frac{R^{2}(u)}{S_{t}}\left(\frac{1}{A}-\frac{1}{u}\right) \\
R(u)=S h^{-1}\left(\sqrt{A^{2}-u^{2}}-\sqrt{A^{2}-1}\right)+1 \text { for } u \in[1 ; A] \\
t_{\text {trans }}=\int_{1}^{u^{s}}(1+k \delta S h) \cdot \frac{u}{A-u} S h^{-1} d u \\
u^{s}=\sqrt{1+2 S h \sqrt{A^{2}-1}-S h^{2}}=\sqrt{A^{2}-\left(\sqrt{A^{2}-1}-S h\right)^{2}}
\end{gathered}
$$

The fiber tip is sharp whenever $S h<\sqrt{A^{2}-1}$, which implies that $u_{s}<A$.

Let us insert eq. (B.7) in eq. (B.9):

$$
t_{\text {trans }}=\int_{1}^{u^{s}} \frac{u}{A-u}\left(S h^{-1}+\delta\left(\frac{1}{u}-\frac{R^{2}(u)}{S_{t}} \frac{A-u}{A u}\right)\right) d u
$$

Developing gives :

$$
t_{\text {trans }}=\int_{1}^{u^{s}}\left(\frac{u S h^{-1}+\delta}{A-u}-\frac{\delta R^{2}(u)}{A S_{t}}\right) d u
$$

This is the sum of two integrals $I_{1}, I_{2}$, where :

$$
I_{1}=\int_{1}^{u^{s}} \frac{u S h^{-1}+\delta}{A-u} d u
$$

and

$$
I_{2}=-\frac{\delta}{A S_{t}} \int_{1}^{u^{s}} R^{2}(u) d u
$$


The first integral is :

$$
I_{1}=-\left(A S h^{-1}+\delta\right) \ln \left(\frac{A-u^{s}}{A-1}\right)-S h^{-1}\left(u^{s}-1\right)
$$

Using the expression of $u^{s}$ yields :

$I_{1}=-\left(\frac{A}{S h}+\delta\right) \ln \left(1-\frac{\sqrt{A^{2}-\left(\sqrt{A^{2}-1}-S h\right)^{2}}-1}{A-1}\right)-\frac{\sqrt{A^{2}-\left(\sqrt{A^{2}-1}-S h\right)^{2}}-1}{S h}$

Inserting eq. (B.8) in eq.(B.14) gives :

$$
I_{2}=-\frac{\delta}{A S_{t} S h^{2}} \int_{1}^{u^{s}}\left(\sqrt{A^{2}-u^{2}}-\sqrt{A^{2}-1}+S h\right)^{2} d u
$$

Developing gives :

$$
I_{2}=-\frac{\delta}{A S_{t} S h^{2}} \int_{1}^{u^{s}}\left(2 A^{2}-1+S h^{2}-2 S h \sqrt{A^{2}-1}-u^{2}+2\left(S h-\sqrt{A^{2}-1}\right) \sqrt{A^{2}-u^{2}}\right) d u
$$

This is decomposed into three terms $I_{21}, I_{22}, I_{23}$. The first one is:

$$
\begin{gathered}
I_{21}=-\frac{\delta}{A S_{t} S h^{2}}\left(2 A^{2}-1+S h^{2}-2 S h \sqrt{A^{2}-1}\right) \int_{1}^{u^{s}} d u \\
I_{21}=-\frac{2 A^{2}-1+S h^{2}-2 S h \sqrt{A^{2}-1}}{A S_{t} S h^{2}}\left(u^{s}-1\right) \delta \\
I_{21}=-\frac{A^{2}+\left(S h-\sqrt{A^{2}-1}\right)^{2}}{A S_{t} S h^{2}}\left(\sqrt{A^{2}-\left(\sqrt{A^{2}-1}-S h\right)^{2}}-1\right) \delta
\end{gathered}
$$

The second one is :

$$
\begin{gathered}
I_{22}=\frac{\delta}{A S_{t} S h^{2}} \int_{1}^{u^{s}} u^{2} d u=\frac{\delta}{3 A S_{t} S h^{2}}\left(\left(u^{s}\right)^{3}-1\right) \\
I_{22}=\frac{\left(A^{2}-\left(\sqrt{A^{2}-1}-S h\right)^{2}\right)^{3 / 2}-1}{3 A S_{t} S h^{2}} \delta
\end{gathered}
$$


The third one is :

$$
\begin{gathered}
I_{23}=-2 \delta \frac{S h-\sqrt{A^{2}-1}}{A S_{t} S h^{2}} \int_{1}^{u^{s}}\left(\sqrt{A^{2}-u^{2}}\right) d u \\
I_{23}=-\delta \frac{S h-\sqrt{A^{2}-1}}{A S_{t} S h^{2}}\left(u^{s} \sqrt{A^{2}-\left(u^{s}\right)^{2}}-\sqrt{A^{2}-1}+A^{2}\left(\arcsin \frac{u^{s}}{A}-\arcsin \frac{1}{A}\right)\right)
\end{gathered}
$$

Noticing that $A^{2}-\left(u^{s}\right)^{2}=\left(\sqrt{A^{2}-1}-S h\right)^{2}$, one has :

$$
\begin{array}{r}
I_{23}=\delta \frac{S h-\sqrt{A^{2}-1}}{A S_{t} S h^{2}}\left(\sqrt{A^{2}-1}-\sqrt{A^{2}-\left(\sqrt{A^{2}-1}-S h\right)^{2}\left(\sqrt{A^{2}-1}-S h\right)+\ldots}\right. \\
\ldots+A^{2}\left(\arcsin \frac{1}{A}-\arcsin \frac{\sqrt{A^{2}-\left(\sqrt{A^{2}-1}-S h\right)^{2}}}{A}\right)(\$ .26)
\end{array}
$$

\section{Appendix C. Integration method to obtain figure 7}

A polynomial fit has been used to integrate equation 21 and plot figure 7 . The curves of figure 6 are parabolic. They can be fitted using a second-order polynomial approximation. Equation (21) rewrites

$$
\frac{d \widetilde{h_{f}}}{d \widetilde{t}}=a+b \widetilde{h_{f}}+c \widetilde{h_{f}^{2}}
$$

where $a, b$, and $c$ are real constants, which are to be determined for each set of values $\left(S h, A, \widetilde{S_{t}} \widetilde{\delta}\right)$. The solution of equation (C.1) is then :

if $4 a c-b^{2}>0$

$$
\widetilde{h_{f}}(\widetilde{t})=\frac{1}{2 c}\left(\Delta \tan \left(\frac{\Delta \widetilde{t}}{2}+\arctan (b / \Delta)\right)-b\right)
$$

with $\Delta=\sqrt{4 a c-b^{2}}$, else

$$
\widetilde{h_{f}}(\widetilde{t})=\frac{1}{2 c}\left(-\Delta \mathcal{R} e\left(\tanh \left(\frac{\Delta \widetilde{t}}{2}+\frac{1}{2} \ln \left[\frac{\Delta-b}{\Delta+b}\right]\right)\right)-b\right)
$$

with $\Delta=\sqrt{-4 a c+b^{2}}$. 